$$
\begin{array}{r}
\text { NXSERDA--9900350/ } \\
\text { New York State Low-Level } \\
\text { Radioactive Waste } \\
\text { Status Report for } 1998
\end{array}
$$

\author{
RECEIVED \\ JUL 15999 \\ OSTI
}

June 1999

DISTRBUTION OF THS DOCUNERT IS UHMUTEO

MASTER

New York State Energy Research and Development Authority 
For more information about the contents of this report, please contact:

John P. Spath, Program Director

Radioactive Waste Policy and

Nuclear Coordination

(518) 862-1090, ext. 3302

jps@nyserda.org
For additional copies of this report, please contact:

New York State Energy Research and Development Authority

286 Washington Avenue Extension

Albany, NY 12203-6399

(518) 862-1090, ext. 3241

amt@nyserda.org

Unless otherwise noted, all data were derived from low-level radioactive waste generator reports received by NYSERDA as of May 4, 1999.

New York State Low-Level Radioactive Waste Status Report for 1998

New York State Energy Research and Development Authority - June 1999 


\section{New York State Low-Level Radioactive Waste Status Report for 1998}

June 1999

New York State Energy Research and Development Authority

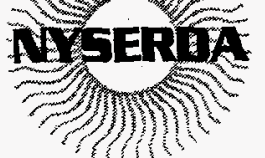


Report preparation: Heidi Voelk

Report presentation: Paula Rosenberg

Unless otherwise noted, all data were derived from low-level radioactive waste generator reports received by NYSERDA as of May 4, 1999.

New York State Low-Level Radioactive Waste Status Report for 1998

New York State Energy Research and Development Authority - June 1999 


\section{DISCLAIMER}

This report was prepared as an account of work sponsored by an agency of the United States Government. Neither the United States Government nor any agency thereof, nor any of their employees, make any warranty, express or implied, or assumes any legal liability or responsibility for the accuracy, completeness, or usefulness of any information, apparatus, product, or process disclosed, or represents that its use would not infringe privately owned rights. Reference herein to any specific commercial product, process, or service by trade name, trademark, manufacturer, or otherwise does not necessarily constitute or imply its endorsement, recommendation, or favoring by the United States Government or any agency thereof. The views and opinions of authors expressed herein do not necessarily state or reflect those of the United States Government or any agency thereof. 


\section{DISCLAIMER}

Portions of this document may be illegible in electronic image products. Images are produced from the best available original document. 


\section{TABLE OF CONTENTS}

Introduction

Section 1: Low-Level Radioactive Waste Shipped for Disposal by

New York State Generators in 1998 3

Table $\quad 1-1 \quad$ Generators Reporting and Shipping Waste for Disposal $\ldots \ldots \ldots \ldots \ldots \ldots \ldots \ldots$

Table $\quad 1-2 \quad$ Volume and Activity of Waste Shipped for Disposal $\ldots \ldots \ldots \ldots \ldots \ldots \ldots \ldots \ldots$

Table $\quad 1-3 \quad$ Waste Shipped for Disposal, by Class and Generator Type $\ldots \ldots \ldots \ldots \ldots \ldots \ldots \ldots$

Table $\quad 1-4 \quad$ Distribution of Waste Among Disposal Facilities . . . . . . . . . . . . . . 7

Table $\quad 1-5 \quad$ Treatments Reported for Waste Shipped for Disposal, by

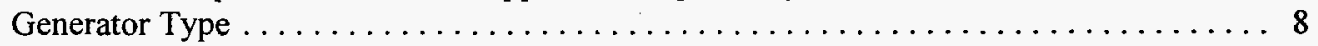

Table $\quad 1-6 \quad$ Number of Facilities Shipping Various Waste Types for Disposal $\ldots \ldots \ldots \ldots \ldots .9$

Table $\quad 1-7 \quad$ Waste Shipped for Disposal, by County of Origin $\ldots \ldots \ldots \ldots \ldots \ldots \ldots \ldots \ldots$

Table $\quad 1-8 \quad$ Radionuclide Content of Waste Shipped for Disposal $\ldots \ldots \ldots \ldots \ldots \ldots \ldots \ldots \ldots$

Table $\quad 1-9 \quad$ Waste Shipped from Various States for Disposal in $1998 \ldots \ldots \ldots \ldots \ldots \ldots \ldots \ldots$

Section 2: Low-Level Radioactive Waste in Storage (as of December 31, 1998) $\ldots \ldots \ldots \ldots \ldots \ldots \ldots 15$

Table $\quad 2-1 \quad$ Generators Reporting and Storing Waste Pending Disposal $\ldots \ldots \ldots \ldots \ldots \ldots \ldots 16$

Table $\quad 2-2 \quad$ Volume and Activity of Waste Stored Pending Disposal $\ldots \ldots \ldots \ldots \ldots \ldots \ldots \ldots$

Table $\quad 2-3 \quad$ Waste in Storage Pending Disposal, by Class and Generator Type $\ldots \ldots \ldots \ldots \ldots 18$

Table $\quad 2-4 \quad$ Number of Facilities Reporting Various Waste Types in Storage Pending Disposal . . . . . . . . . . . . . . . . . . . . . . . 19

Table $\quad 2-5 \quad$ Waste in Storage Pending Disposal, by County of Origin $\ldots \ldots \ldots \ldots \ldots \ldots \ldots \ldots$

Table $\quad 2-6 \quad$ Radionuclide Content of Waste in Storage Pending Disposal $\ldots \ldots \ldots \ldots \ldots \ldots \ldots 22$

Table $\quad 2-7 \quad$ Waste Reported in Storage for Decay, by Generator Type . . . . . . . . . . . . . . 24

Table $\quad 2-8 \quad$ Treatments Reported for Waste in Storage Pending Disposal, by Generator Type . . . . 25

\section{Section 3: Historic Data and Projections for Low-Level Radioactive Waste}

Generation in New York State

Table $\quad 3-1 \quad$ Historic Overview of Waste Disposal, by Volume $\ldots \ldots \ldots \ldots \ldots \ldots \ldots \ldots \ldots$

Table $\quad 3-2 \quad$ Historic Overview of Waste Disposal, by Activity $\ldots \ldots \ldots \ldots \ldots \ldots \ldots \ldots \ldots \ldots$

Figure $\quad 3-1 \quad$ Historic Overview of Waste Disposal, by Volume $\ldots \ldots \ldots \ldots \ldots \ldots \ldots \ldots \ldots$

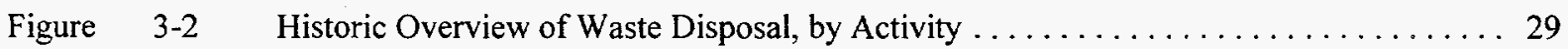

Table $\quad 3-3 \quad$ Generators' Five-Year Projections of Waste, by Volume $\ldots \ldots \ldots \ldots \ldots \ldots \ldots \ldots$

Table $\quad 3-4 \quad$ Generators' Five-Year Projections of Waste, by Activity $\ldots \ldots \ldots \ldots \ldots \ldots \ldots \ldots$ 
Section 4: Generators Filing Reports

Table $\quad 4$-a Generators Reporting Disposal or Storage of Waste Pending Disposal . . . . . . . . 32

Table $\quad 4-b \quad$ Generators Reporting Only Storage for Decay $\ldots \ldots \ldots \ldots \ldots \ldots \ldots \ldots \ldots \ldots$

Conversions for Units $\ldots \ldots \ldots \ldots \ldots \ldots \ldots \ldots \ldots \ldots \ldots \ldots \ldots \ldots \ldots \ldots \ldots \ldots \ldots \ldots \ldots$ Inside Back Cover 


\section{INTRODUCTION}

This report summarizes data on low-level radioactive waste (LLRW) generated in New York State. ${ }^{2}$ It is based on reports from generators that must be filed annually with the New York State Energy Research and Development Authority (NYSERDA) and on data from the U.S. Department of Energy (U.S. DOE).

The New York State Low-Level Radioactive Waste Management Act (State Act) requires LLRW generators in the State to submit annual reports detailing the classes and quantities of waste generated. This is the 13 th year generators have been required to submit these reports to NYSERDA.

The data are summarized in a series of tables and figures. There are four sections in the report. Section 1 covers volume, activity, and other characteristics of waste shipped for disposal in 1998. (Activity is the measure of a material's radioactivity, or the number of radiation-emitting events occurring each second.) Section 2 summarizes volume, activity, and other characteristics of waste held for storage as of December 31, 1998. Section 3 shows historical LLRW generation and includes generators' projections for the next five years. Section 4 provides a list, by county, of all facilities from which 1998 LLRW reports were received.

Volume is presented in cubic meters and activity is presented in gigabecquerels (GBq) or megabecquerels (MBq). These units have been adopted to be consistent with U.S. Nuclear Regulatory Commission uniform national LLRW manifest requirements. The Conversions for Units table on the inside back cover and footnotes to the relevant tables provide information for converting the data to the previously used units of cubic feet and curies.

1 Low-level radioactive waste is one category of waste produced through processes that use radioactive materials. In the U.S., radioactive wastes are classified according to a number of different categories by federal law and U.S. Nuclear Regulatory Commission regulations.

2 Waste generated by certain federal installations or programs, such as the Brookhaven National Laboratory, the Knolls Atomic Power Laboratory, and the West Valley Demonstration Project, are not included in this report or in the requirements for generator reporting to NYSERDA. Under the federal Low-Level Radioactive Waste Policy Act as amended in 1985. (Public Law 99-240), the federal government, not the states, is responsible for disposal of LLRW owned or generated by the U.S. DOE, the U.S. Navy as a result of decommissioning vessels, or the federal government as a result of research, development, testing, or production of nuclear weapons. 


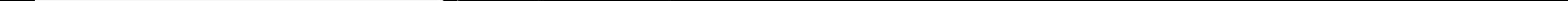




\section{Section 1}

\section{LOW-LEVEL RADIOACTIVE WASTE SHIPPED FOR DISPOSAL BY NEW YORK STATE GENERATORS IN 1998}

This section summarizes data reported by LLRW generators in New York State on waste transferred to licensed LLRW disposal facilities in Barnwell, South Carolina; Clive, Utah (Envirocare); and Richland, Washington, during 1998.

While the Barnwell facility will accept most types of LLRW, the Clive and Richland facilities are more restrictive. While the Clive facility can accept most Class A waste, it cannot accept Class B or C waste. It can also accept, treat, and dispose of most solid, mixed waste (i.e., LLRW that also contains hazardous chemicals) that meets the site's radioactivity concentration limits. The Richland facility is authorized to accept limited volumes of LLRW containing small quantities of naturally occurring radioactive material (e.g., uranium, thorium) from New York State generators.

Volume is presented in cubic meters and activity is presented in gigabecquerels (GBq) or megabecquerels (MBq). These units have been adopted to be consistent with U.S. Nuclear Regulatory Commission uniform national LLRW manifest requirements. The Conversions for Units table on the inside back cover and footnotes to the relevant tables provide information for converting the data to the previously used units of cubic feet and curies.

In 1998, generators in New York State reported disposing of 354 cubic meters (12,510 cubic feet) of LLRW containing $2,023,092 \mathrm{GBq}(54,678$ curies) of radioactivity. About $15 \%$ of the volume of LLRW, containing less than $1 \%$ of the radioactivity, was shipped to the Clive, Utah, facility. The Barnwell, South Carolina, facility received $85 \%$ of the volume and more than $99 \%$ of the radioactivity.

Individual entries in the following tables have been rounded using standard procedures. Because the totals shown represent the sum of the rounded entries, they may vary slightly from one table to another and may not always equal $100 \%$. Waste volumes have been rounded to the nearest 10th of a cubic meter. In most cases, activity has been rounded to the nearest 10,000th of a GBq. Percentages have been rounded to the nearest 10th of a percent in the tables and figures. 
Table 1-1: Generators Reporting and Shipping Waste for Disposal ${ }^{1}$

\begin{tabular}{|c|c|c|}
\hline Generator Type & Number Reporting & Number $^{2}$ Shipping \\
\hline \multicolumn{3}{|l|}{ MEDICAL } \\
\hline Government & 21 & 4 \\
\hline Private & 210 & 6 \\
\hline College & 20 & 13 \\
\hline Other & 5 & 0 \\
\hline Total Medical & 256 & 23 \\
\hline
\end{tabular}

INDUSTRIAL

Manufacturing

10

4

Research \& Development

9

Other

0

Total Industrial

ACADEMIC (non-medical)

College or University

32

9

Other

Total Academic

GOVERNMENT (non-medical)

New York State

2

2

Other

6

2

Total Government

8

4

TOTAL NON-POWER PLANT

328

50

NUCLEAR POWER PLANT

6

6

TOTAL

334

56

I In addition to shipping LLRW for disposal, other waste management methods include storage of LLRW pending disposal and storage for decay. Section 2 provides information on stored LLRW.

2 Refers to the number of generators who reported transferring LLRW either directly or via a broker or processor to one of the available licensed LLRW disposal facilities. as of May 4, 1999. 
Table 1-2: Volume and Activity of Waste Shipped for Disposal ${ }^{1}$

\begin{tabular}{|c|c|c|c|c|}
\hline Generator Type & $\begin{array}{c}\text { Volume } \\
\left(\mathbf{m}^{3}\right)^{2}\end{array}$ & $\begin{array}{l}\% \text { of } \\
\text { Total } \\
\end{array}$ & $\begin{array}{r}\text { Activity } \\
(\mathbf{G B q})^{2} \\
\end{array}$ & $\begin{array}{l}\% \text { of } \\
\text { Total }\end{array}$ \\
\hline \multicolumn{5}{|l|}{ MEDICAL } \\
\hline Government & 1.0 & & 10.6323 & \\
\hline Private & 16.0 & & 22.0977 & \\
\hline College & 34.6 & & 69.9981 & \\
\hline Total Medical & 51.6 & 14.6 & 102.7281 & * \\
\hline
\end{tabular}

INDUSTRIAL

Manufacturing

8.0

1.0201

Research \& Development

7.0

64.0337

Total Industrial

15.0

4.2

65.0538

ACADEMIC (non-medical)

College or University

10.6

63.3536

Other

0.6

0.1110

Total Academic

11.2

3.2

63.4646

GOVERNMENT (non-medical)

New York State

0.7

0.7315

Other

2.9

0.5566

Total Government

3.6

1.0

1.2881

TOTAL NON-POWER PLANT

81.4

23.0

232.5346

NUCLEAR POWER PLANT

272.9

77.0

$2,022,859.4769$

100.0

TOTAL

354.3

100.0

$2,023,092.0115$

100.0

$\left(12,510 \mathrm{ft}^{3}\right)$

(54,678 curies)

1 Refers to LLRW transferred either directly or via a broker or processor to one of the available licensed LLRW disposal facilities.

2. To obtain volume in cubic feet, multiply the number of cubic meters by 35.31 . To obtain activity in curies, divide the number of gigabecquerels $(\mathrm{GBq})$ by 37 .

* Less than $0.1 \%$. as of May 4, 1999. 
Table 1-3: Waste Shipped ${ }^{1}$ for Disposal, by Class ${ }^{2}$ and Generator Type

\begin{tabular}{|c|c|c|c|c|c|c|}
\hline \multirow[b]{2}{*}{ Generator Type } & \multicolumn{2}{|c|}{ Class A } & \multicolumn{2}{|c|}{ Class B } & \multicolumn{2}{|c|}{ Class C } \\
\hline & $\begin{array}{c}\text { Volume } \\
\left(\mathrm{m}^{3}\right)^{3}\end{array}$ & $\begin{array}{l}\text { Activity } \\
(\mathbf{G B q})^{3}\end{array}$ & $\begin{array}{c}\text { Volume } \\
\left(\mathbf{m}^{3}\right)^{3}\end{array}$ & $\begin{array}{l}\text { Activity } \\
(\mathbf{G B q})^{3}\end{array}$ & $\begin{array}{c}\text { Volume } \\
\left(\mathrm{m}^{3}\right)^{3}\end{array}$ & $\begin{array}{r}\text { Activity } \\
(\mathbf{G B q})^{3}\end{array}$ \\
\hline MEDICAL & 51.6 & 102.6081 & 0.0 & 0.0000 & $*$ & 0.1200 \\
\hline \multicolumn{7}{|l|}{ INDUSTRIAL } \\
\hline Manufacturing & 8.0 & 1.0201 & 0.0 & 0.0000 & 0.0 & 0.0000 \\
\hline Research \& Development & 6.9 & 57.5588 & 0.0 & 0.0000 & 0.1 & 6.4750 \\
\hline ACADEMIC & 11.2 & 63.4646 & 0.0 & 0.0000 & 0.0 & 0.0000 \\
\hline GOVERNMENT & 3.6 & 0.7331 & $*$ & 0.5550 & 0.0 & 0.0000 \\
\hline $\begin{array}{l}\text { NUCLEAR POWER } \\
\text { PLANT }\end{array}$ & 193.5 & $24,151.6870$ & 35.7 & $17,322.7407$ & 43.7 & $1,981,385.0491$ \\
\hline TOTAL & $\begin{array}{c}274.8 \\
\left(9,703 \mathrm{ft}^{3}\right)\end{array}$ & $\begin{array}{l}24,377.0717 \\
(659 \text { curies })\end{array}$ & $\begin{array}{c}35.7 \\
\left(1,261 \mathrm{ft}^{3}\right)\end{array}$ & $\begin{array}{l}17,323.2957 \\
(468 \text { curies })\end{array}$ & $\begin{array}{r}43.8 \\
\left(1,546 \mathrm{ft}^{3}\right)\end{array}$ & $\begin{array}{l}1,981,391.6441 \\
(53,551 \text { curies })\end{array}$ \\
\hline \multicolumn{7}{|c|}{$\begin{array}{l}2 \text { Classes A, B, and C are waste-classification categories established by the U.S. Nuclear Regulatory Commission (NRC) in Title } 10 \text { of the } \\
\text { Code of Federal Regulations, Part } 61 \text {, "Licensing Requirements for Land Disposal of Radioactive Waste," and adopted by the New York State } \\
\text { Department of Environmental Conservation in } 6 \text { NYCRR Part 382, "Regulations for Low-Level Radioactive Waste Disposal Facilities." }\end{array}$} \\
\hline \multicolumn{7}{|c|}{$\begin{array}{l}\text { To obtain volume in cubic feet, multiply the number of cubic meters by } 35.31 \text {. To obtain activity in curies, divide the number of } \\
\text { gigabecquerels }(\mathrm{GBq}) \text { by } 37 \text {. }\end{array}$} \\
\hline
\end{tabular}

Unless otherwise noted, all data were derived from low-level radioactive waste generator reports received by NYSERDA as of May 4, 1999. 
Table 1-4: Distribution of Waste Among Disposal Facilities ${ }^{1}$

\begin{tabular}{|c|c|c|c|c|}
\hline Disposal Facility & $\begin{array}{c}\text { Volume } \\
\left(\mathrm{m}^{3}\right)^{2}\end{array}$ & $\%$ of Total & $\begin{array}{l}\text { Activity } \\
(\mathbf{G B q})^{2}\end{array}$ & $\%$ of Total \\
\hline Barnwell, South Carolina & 301.8 & 85.2 & $2,023,078.4057$ & 99.9 \\
\hline Clive, Utah & 52.3 & 14.8 & 13.6056 & $*$ \\
\hline Richland, Washington & 0.2 & $*$ & 0.0002 & * \\
\hline TOTAL & $\begin{array}{r}354.3 \\
\left(12,510 \mathrm{ft}^{3}\right)\end{array}$ & 100.0 & $\begin{array}{l}2,023,092.0115 \\
(54,678 \text { curies })\end{array}$ & 100.0 \\
\hline
\end{tabular}

Refers to LLRW transferred either directly or via a broker or processor to the respective disposal facility.

2 To obtain volume in cubic feet, multiply the number of cubic meters by 35.31. To obtain activity in curies, divide the number of gigabecquerels $(\mathrm{GBq})$ by 37 .

* Less than $0.1 \%$.

Unless otherwise noted, all data were derived from low-level radioactive waste generator reports received by NYSERDA as of May 4, 1999. 


\begin{tabular}{|c|c|c|}
\hline Generator Type & $\begin{array}{c}\text { Number of Generators } \\
\text { Shipping LLRW }\end{array}$ & $\begin{array}{c}\text { Number of Shipping Generators Reporting Waste } \\
\text { Treatment and Predominant Treatments }\end{array}$ \\
\hline \multirow[t]{2}{*}{ MEDICAL } & 23 & $\begin{aligned} \text { On Site: } & 16 \\
& \cdot \text { Compaction } \\
& \text { - Solidification }\end{aligned}$ \\
\hline & & $\begin{aligned} \text { Off Site: } & 9 \\
& - \text { Supercompaction } \\
& \cdot \text { Incineration }\end{aligned}$ \\
\hline \multirow[t]{2}{*}{ INDUSTRIAL } & 13 & $\begin{aligned} \text { On Site: } & 4 \\
& \text { - Compaction } \\
& \cdot \text { Solidification }\end{aligned}$ \\
\hline & & $\begin{aligned} \text { Off Site: } & 8 \\
& - \text { Supercompaction } \\
& \cdot \text { Incineration }\end{aligned}$ \\
\hline
\end{tabular}

ACADEMIC
10
On Site: 4

- Compaction

- Solidification

Off Site: 8

- Supercompaction

- Incineration

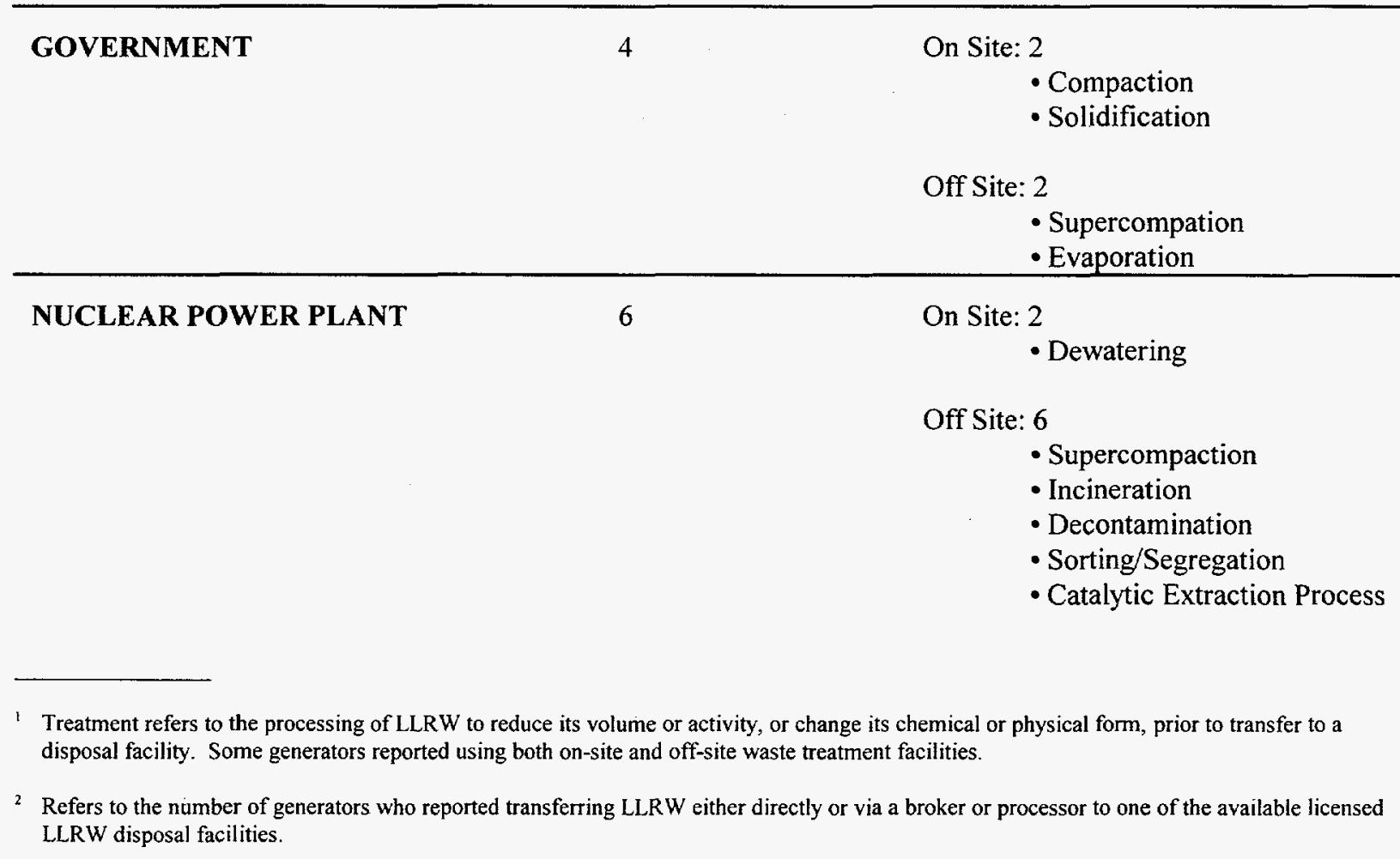

Unless otherwise noted, all data were derived from low-level radioactive waste generator reports received by NYSERDA as of May 4, 1999. 


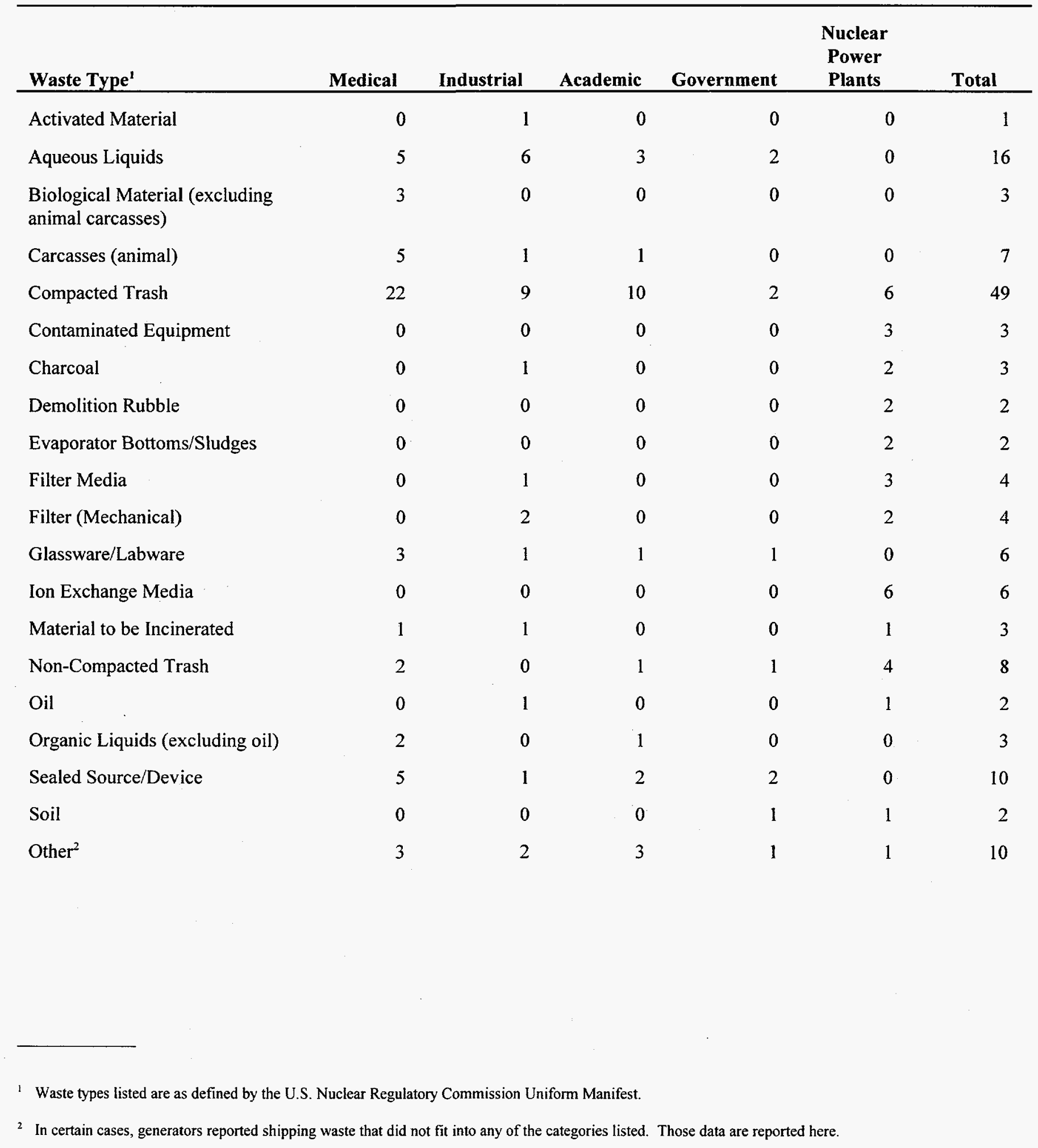


Table 1-7: Waste Shipped for Disposal, by County of Origin ${ }^{1}$

\begin{tabular}{|c|c|c|c|c|c|c|}
\hline County & $\begin{array}{c}\text { Number of } \\
\text { Generators } \\
\text { Reporting } \\
\end{array}$ & $\begin{array}{c}\text { Number of } \\
\text { Generators } \\
\text { Shipping LLRW } \\
\end{array}$ & $\begin{array}{c}\text { Volume } \\
\left(\mathrm{m}^{3}\right)^{3}\end{array}$ & $\begin{array}{l}\% \text { of } \\
\text { Total }\end{array}$ & $\begin{array}{l}\text { Activity } \\
{\text { (GBq })^{3}}^{3}\end{array}$ & $\begin{array}{l}\% \text { of } \\
\text { Total }\end{array}$ \\
\hline Albany & 11 & 3 & 4.2 & 1.2 & 2.6589 & * \\
\hline Allegany & 0 & 0 & 0.0 & 0.0 & 0.0000 & 0.0 \\
\hline Bronx & 12 & 2 & 0.1 & $*$ & 7.0894 & $*$ \\
\hline Broome & 6 & 1 & $*$ & 0.0 & 0.0224 & $*$ \\
\hline Cattaraugus & 1 & 1 & $*$ & 0.0 & 0.0128 & * \\
\hline Cayuga & 1 & 0 & 0.0 & 0.0 & 0.0000 & 0.0 \\
\hline Chautauqua & 2 & 0 & 0.0 & 0.0 & 0.0000 & 0.0 \\
\hline Chemung & 4 & 1 & 0.4 & 0.1 & 0.0857 & $*$ \\
\hline Chenango & 0 & 0 & 0.0 & 0.0 & 0.0000 & 0.0 \\
\hline Clinton & 4 & 2 & 0.2 & 0.1 & 3.1820 & $*$ \\
\hline Columbia & 0 & 0 & 0.0 & 0.0 & 0.0000 & 0.0 \\
\hline Cortland & 1 & 0 & 0.0 & 0.0 & 0.0000 & 0.0 \\
\hline Delaware & 2 & 0 & 0.0 & 0.0 & 0.0000 & 0.0 \\
\hline Dutchess & 8 & 1 & 0.1 & $*$ & 0.0029 & $*$ \\
\hline Erie & 20 & 2 & 8.6 & 2.4 & 12.6530 & $*$ \\
\hline Essex & 3 & 1 & 0.1 & $*$ & 0.0021 & $*$ \\
\hline Franklin & 2 & 0 & 0.0 & 0.0 & 0.0000 & 0.0 \\
\hline Fulton & 0 & 0 & 0.0 & 0.0 & 0.0000 & 0.0 \\
\hline Genesee & 1 & 0 & 0.0 & 0.0 & 0.0000 & 0.0 \\
\hline Greene & 0 & 0 & 0.0 & 0.0 & 0.0000 & 0.0 \\
\hline Hamilton & 0 & 0 & 0.0 & 0.0 & 0.0000 & 0.0 \\
\hline Herkimer & 1 & 0 & 0.0 & 0.0 & 0.0000 & 0.0 \\
\hline Jefferson & 3 & 0 & 0.0 & 0.0 & 0.0000 & 0.0 \\
\hline Kings & 18 & 2 & 2.5 & 0.7 & 0.1059 & * \\
\hline Lewis & 0 & 0 & 0.0 & 0.0 & 0.0000 & 0,0 \\
\hline Livingston & 1 & 0 & 0.0 & 0.0 & 0.0000 & 0.0 \\
\hline Madison & 2 & 0 & 0.0 & 0.0 & 0.0000 & 0.0 \\
\hline Monroe & 12 & 2 & 8.2 & 2.3 & 42.4332 & * \\
\hline Montgomery & 2 & 0 & 0.0 & 0.0 & 0.0000 & 0.0 \\
\hline Nassau & 34 & 4 & 5.0 & 1.4 & 2.2035 & $*$ \\
\hline New York & 32 & 11 & 25.7 & 7.2 & 74.1764 & $*$ \\
\hline Niagara & 2 & 1 & 0.2 & 0.1 & 0.0010 & $*$ \\
\hline Oneida & 4 & 0 & 0.0 & 0.0 & 0.0000 & 0.0 \\
\hline Onondaga & 11 & 2 & 0.6 & 0.2 & 6.6949 & $*$ \\
\hline
\end{tabular}

Unless otherwise noted, all data were derived from low-level radioactive waste generator reports received by NYSERDA as of May 4, 1999. 
Table 1-7: Waste Shipped for Disposal, by County of Origin ${ }^{1}$ (continued)

\begin{tabular}{|c|c|c|c|c|c|c|}
\hline County & $\begin{array}{c}\text { Number of } \\
\text { Generators } \\
\text { Reporting } \\
\end{array}$ & $\begin{array}{c}\text { Number of } \\
\text { Generators } \\
\text { Shipping LLRW } \\
\end{array}$ & $\begin{array}{l}\text { Volume } \\
\left(\mathrm{m}^{3}\right)^{3}\end{array}$ & $\begin{array}{l}\% \text { of } \\
\text { Total }\end{array}$ & $\begin{array}{l}\text { Activity } \\
(\mathbf{G B q})^{3}\end{array}$ & $\begin{array}{l}\% \text { of } \\
\text { Total }\end{array}$ \\
\hline Ontario & 3 & 0 & 0.0 & 0.0 & 0.0000 & 0.0 \\
\hline Orange & 8 & 0 & 0.0 & 0.0 & 0.0000 & 0.0 \\
\hline Orleans & 1 & 0 & 0.0 & 0.0 & 0.0000 & 0.0 \\
\hline Oswego & 3 & 3 & 164.7 & 46.5 & $1,910,519.5262$ & 94.4 \\
\hline Otsego & 3 & 0 & 0.0 & 0.0 & 0.0000 & 0.0 \\
\hline Putnam & 2 & 0 & 0.0 & 0.0 & 0.0000 & 0.0 \\
\hline Queens & 13 & 1 & 0.2 & 0.1 & 1.6939 & $*$ \\
\hline Rensselaer & 4 & 1 & 2.1 & 0.6 & 0.2634 & $*$ \\
\hline Richmond & 5 & 0 & 0.0 & 0.0 & 0.0000 & 0.0 \\
\hline Rockland & 8 & 3 & 1.1 & 0.3 & 56.4719 & $*$ \\
\hline St. Lawrence & 3 & 0 & 0.0 & 0.0 & 0.0000 & 0.0 \\
\hline Saratoga & 3 & 0 & 0.0 & 0.0 & 0.0000 & 0.0 \\
\hline Schenectady & 4 & 1 & 1.1 & 0.3 & 1.0981 & $*$ \\
\hline Schoharie & 0 & 0 & 0.0 & 0.0 & 0.0000 & 0.0 \\
\hline Schuyler & 0 & 0 & 0.0 & 0.0 & 0.0000 & 0.0 \\
\hline Seneca & 1 & 1 & 2.9 & 0.8 & 0.0016 & $*$ \\
\hline Steuben & 2 & 0 & 0.0 & 0.0 & 0.0000 & 0.0 \\
\hline Suffolk & 33 & 1 & 3.6 & 1.0 & 1.8305 & $*$ \\
\hline Sullivan & 0 & 0 & 0.0 & 0.0 & 0.0000 & 0.0 \\
\hline Tioga & 0 & 0 & 0.0 & 0.0 & 0.0000 & 0.0 \\
\hline Tompkins & 3 & 1 & 0.8 & 0.2 & 3.9351 & $*$ \\
\hline Ulster & 6 & 0 & 0.0 & 0.0 & 0.0000 & 0.0 \\
\hline Warren & 1 & 0 & 0.0 & 0.0 & 0.0000 & 0.0 \\
\hline Washington & 0 & 0 & 0.0 & 0.0 & 0.0000 & 0.0 \\
\hline Wayne & 2 & 1 & 15.6 & 4.4 & $7,319.1620$ & 0.4 \\
\hline Westchester & 26 & 7 & 106.5 & 30.1 & $105,036.7059$ & 5.2 \\
\hline Wyoming & 0 & 0 . & 0.0 & 0.0 & 0.0000 & 0.0 \\
\hline Yates & 0 & 0 & 0.0 & 0.0 & 0.0000 & 0.0 \\
\hline TOTALS & 334 & 56 & $\begin{array}{c}354.3 \\
\left(12,510 \mathrm{ft}^{3}\right)\end{array}$ & 100.0 & $\begin{array}{r}2,023,092.0115 \\
(54,678 \text { curies })\end{array}$ & 100.0 \\
\hline $\begin{array}{l}\text { Section } 4 \text { of this } \\
\text { Refers to the nun } \\
\text { licensed LLRW } \\
\text { To obtain volum } \\
\text { gigabecquerels ( } \\
\text { Less than } 0.1 \text { cut }\end{array}$ & $\begin{array}{l}\text { lentifies the indiv } \\
\text { generators that re } \\
\text { facilities. } \\
\text { c feet, multiply tl } \\
37 \text {. } \\
\text { r or } 0.1 \%\end{array}$ & $\begin{array}{l}\text { ual facilities reporting an } \\
\text { tred transferring LLRW e } \\
\text { number of cubic meters }\end{array}$ & $\begin{array}{l}\text { d shipping waste } \\
\text { either directly or } \\
\text { by } 35.31 \text {. To obt }\end{array}$ & $\begin{array}{l}\text { or disposal. } \\
\text { a a broker or } \\
\text { in activity in }\end{array}$ & $\begin{array}{l}\text { processor to one of the available } \\
\text { curies, divide the number of }\end{array}$ & \\
\hline
\end{tabular}

Unless otherwise noted, all data were derived from low-level radioactive waste generator reports received by NYSERDA as of May 4, 1999. 
Table 1-8: Radionuclide Content of Waste ${ }^{1}$ Shipped for Disposal (in MBq) ${ }^{2}$

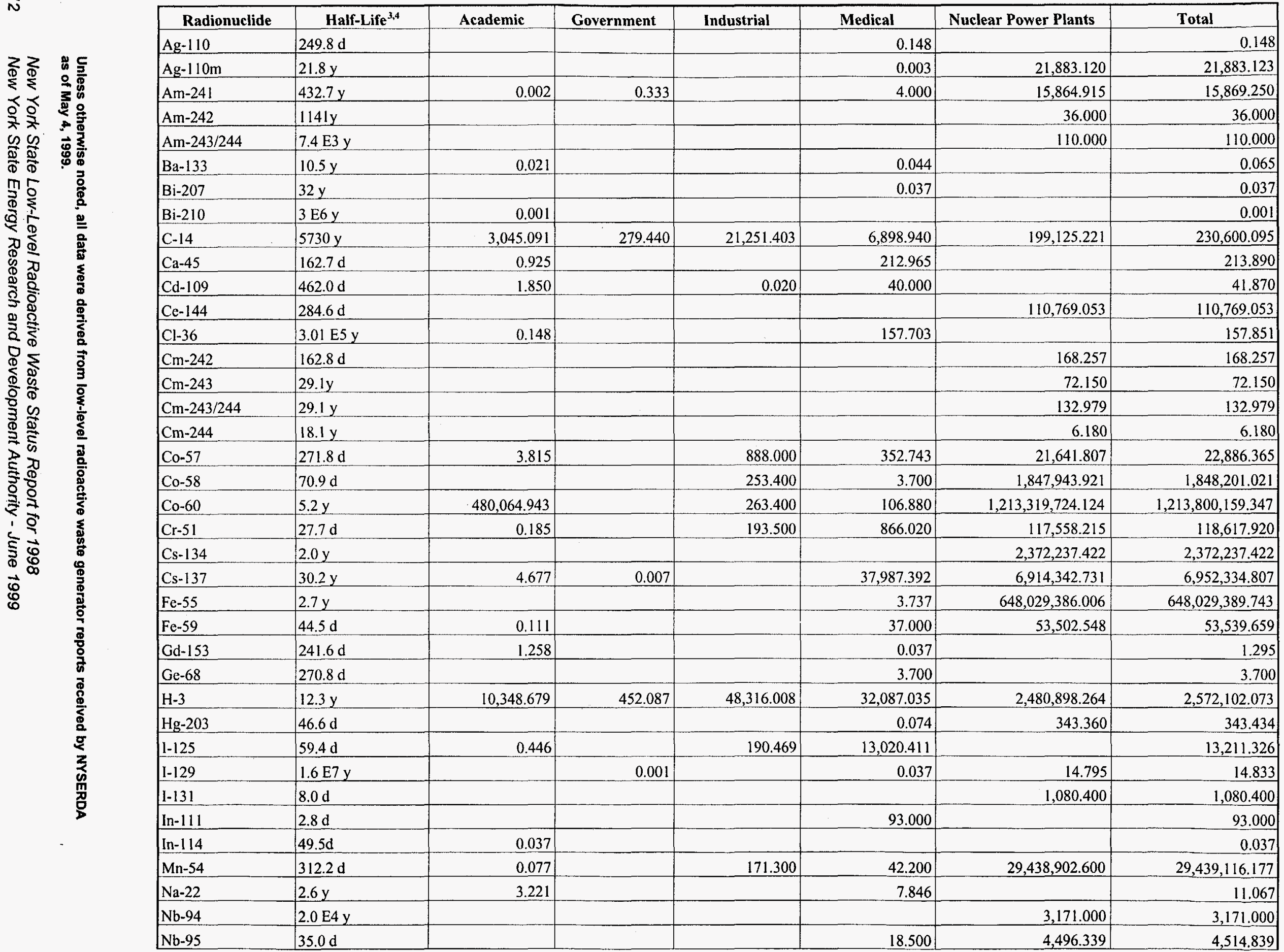




\begin{tabular}{|c|c|c|c|c|c|c|c|}
\hline Ni-59 & $7.6 \mathrm{E} 4 \mathrm{y}$ & & & & & $885,870.650$ & $885,870.650$ \\
\hline $\mathrm{Ni}-63$ & $100 y$ & $1,908.460$ & 555.000 & 370.000 & 113.300 & $110,721,098.183$ & $110,724,044.943$ \\
\hline P-32 & $14.3 \mathrm{~d}$ & & & 82.259 & 469.900 & & 552.159 \\
\hline P-33 & $25.3 d$ & & & 517.325 & 25.000 & & 542.325 \\
\hline Pm-147 & $2.6 \mathrm{y}$ & 0.001 & & & & & 0.001 \\
\hline $\mathrm{Pu}-238$ & $87.7 y$ & & & & & 287.419 & 287.419 \\
\hline $\mathrm{Pu}-239$ & $2.4 \mathrm{E} 4 \mathrm{y}$ & 0.002 & & & & 236.020 & 236.022 \\
\hline $\mathrm{Pu}-239 / 240$ & $2.4 \mathrm{E} 4 \mathrm{y}$ & & & & & 345.278 & 345.278 \\
\hline $\mathrm{Pu}-241$ & $14.4 \mathrm{y}$ & & & & & $102,114.855$ & $102,114.855$ \\
\hline $\mathrm{Ra}-226$ & $1.6 \mathrm{E} 3 \mathrm{y}$ & & 1.110 & & 8.400 & & 9.510 \\
\hline $\mathrm{Rb}-86$ & $18.7 \mathrm{~d}$ & & & & 43.000 & & 43.000 \\
\hline $\mathrm{Re}-186$ & 2 E5 y & 0.002 & & & & & 0.002 \\
\hline Ru-103 & $39.3 \mathrm{~d}$ & & & & 18.500 & 59.000 & 77.500 \\
\hline Ru-106 & $1 y$ & & & & & $31,524.000$ & $31,524.000$ \\
\hline S-35 & $87.2 \mathrm{~d}$ & 0.004 & & 268.159 & $3,503.646$ & & $3,771.809$ \\
\hline Sb-124 & $60.2 \mathrm{~d}$ & & & & & $7,568.360$ & $7,568.360$ \\
\hline $\mathrm{Sb}-125$ & $2.8 \mathrm{y}$ & & & & & $502,142.118$ & $502,142.118$ \\
\hline $\mathrm{Sc}-46$ & $83.8 \mathrm{~d}$ & 0.002 & & & 18.537 & & 18.539 \\
\hline $\mathrm{Se}-75$ & $119.8 \mathrm{~d}$ & & & & 40.000 & & 40.000 \\
\hline Sn-113. & $115.1 \mathrm{~d}$ & 0.178 & & & 18.500 & $1,608.090$ & $1,626.768$ \\
\hline $\mathrm{Sn}-117 \mathrm{~m}$ & $13.6 \mathrm{~d}$ & & & & & 27.000 & 27.000 \\
\hline Sr-89 & $50.5 \mathrm{~d}$ & & & & & 821.128 & 821.128 \\
\hline $\mathrm{Sr}-90$ & $29.1 \mathrm{y}$ & & & & $6,321.450$ & $28,845.718$ & $35,167.168$ \\
\hline Tc-99 & 2.1 E5 y & 43.800 & 0.002 & & & $2,735.700$ & $2,779.502$ \\
\hline Tc-99m & $6.0 \mathrm{~h}$ & & & 18.500 & & & 18.500 \\
\hline Th-232 & $1.4 \mathrm{E} 10 \mathrm{y}$ & 4.446 & & 4.800 & 17.679 & & 26.925 \\
\hline Tl-204 & $3.8 \mathrm{y}$ & & & & 0.010 & & 0.010 \\
\hline $\mathrm{U}-235$ & $7 \mathrm{E} 8 \mathrm{y}$ & & & 6.330 & & & 6.330 \\
\hline $\mathrm{U}-238$ & $4.5 \mathrm{Eg} \mathrm{y}$ & 31.990 & 0.074 & 5.480 & 145.479 & & 183.023 \\
\hline $\mathrm{Zn}-65$ & $243.8 \mathrm{~d}$ & 0.330 & & 209.800 & 37.700 & $5,174,502.941$ & $5,174,750.771$ \\
\hline $\mathrm{Zr}-95$ & $64 \mathrm{~d}$ & & & & & $6,325.596$ & $6,325.596$ \\
\hline Others $^{5}$ & & & & & 1.000 & & 1.000 \\
\hline \multicolumn{2}{|l|}{ Total } & $495,464.702$ & $1,288.054$ & $73,010.153$ & $102,725.105$ & $2,022,419,523.463$ & $2,023,092,011.477$ \\
\hline
\end{tabular}

Some generator facilities have reported radionuclides with half-lives of less than 90 days in LLRW shipped for disposal. In the majority of these cases, the shorter-lived radionuclides reported cannot be separated readily from longer-lived radionuclides in the waste.

2 To obtain activity in curies, divide the number of $\mathrm{MBq}$ by 37,000 .

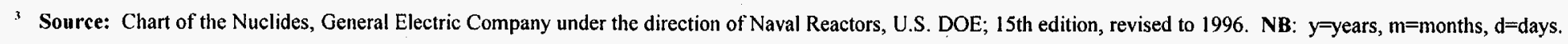

4 Where scientific notation is used, multiply the number by 10 to the specified power. Example: For Ra-226, $1.6 \mathrm{E} 3=1.6 \times 10^{3}=1.6 \times 10 \times 10 \times 10=1.6 \times 1,000=1,600$. For this table, the whole number to which 10 is raised is equal to the number of places the decimal is moved to the right.

5 In certain cases, LLRW generators are permitted by manifest regulations to report a single activity for a group of radionuclides without assigning a value to each; those data are reported here. 
Table 1-9: Waste Shipped from Various States for Disposal in 1998

\begin{tabular}{|c|c|c|c|}
\hline State & Cubic Meters of Waste ${ }^{2}$ & State & Cubic Meters of Waste \\
\hline Tennessee & $12,188.7$ & Nebraska & 81.8 \\
\hline Unknown $^{3}$ & $4,401.3$ & Colorado & 51.4 \\
\hline Massachusetts & $4,375.7$ & Hawaii & 50.8 \\
\hline Ohio & $3,665.3$ & Louisana & 49.1 \\
\hline Oregon & $2,596.8$ & Wisconsin & 43.6 \\
\hline Michigan & $2,320.9$ & Minnesota & 38.9 \\
\hline Illinois & $1,820.4$ & Iowa & 29.1 \\
\hline Pennsylvania & $1,196.9$ & Kansas & 28.4 \\
\hline Florida & $1,108.9$ & Oklahoma & 22.3 \\
\hline Washington & 862.7 & Mississippi & 21.8 \\
\hline Virginia & 693.2 & New Mexico & 11.2 \\
\hline Utah & 481.7 & Arkansas & 10.2 \\
\hline Missouri & 473.2 & New Hampshire & 7.4 \\
\hline Texas & 432.8 & District of Columbia & 6.9 \\
\hline South Carolina & 396.8 & Delaware & 4.9 \\
\hline California & 391.3 & Indiana & 2.4 \\
\hline New York ${ }^{4}$ & 387.4 & Alaska & 2.3 \\
\hline Arizona & 329.0 & Rhode Island & 1.8 \\
\hline Georgia & 290.5 & Nevada & 1.6 \\
\hline New Jersey & 253.4 & North Dakota & 1.4 \\
\hline Connecticut & 242.5 & West Virginia & 1.3 \\
\hline North Carolina & 218.5 & Vermont & 0.7 \\
\hline Maryland & 213.1 & Montana & 0.3 \\
\hline Alabama & 212.1 & Wyoming & 0.2 \\
\hline Maine & 118.5 & Idaho & 0.2 \\
\hline \multirow[t]{2}{*}{ Kentucky } & 84.3 & South Dakota & 0.1 \\
\hline & & Total & $40,226.0$ \\
\hline \multicolumn{4}{|c|}{$\begin{array}{l}\text { This information is obtained from the U.S. DOE Manifest Information Management System (MIMS) database, as of April } 30,1999 . \\
\text { The data represent LLRW shipped to the Richland, Washington; Clive, Utah (Envirocare); and Barnwell, South Carolina, disposal facilities. } \\
\text { Envirocare's disposal data do not see through waste processors. Envirocare assigns waste received from waste processors to the State in which } \\
\text { the waste processor is located, not the State in which the waste was generated. To the reader, this means that the state reflected in MIMS, for } \\
\text { waste received at Envirocare, may not be the state in which the waste was generated. }\end{array}$} \\
\hline \multicolumn{4}{|c|}{ To obtain volume in cubic feet, multiply the number of cubic meters by 35.31 . } \\
\hline Waste reported to th & by Envirocare that was not ass & f origin. & \\
\hline
\end{tabular}




\section{Section 2}

\section{LOW-LEVEL RADIOACTIVE WASTE IN STORAGE (as of December 31, 1998)}

This section provides information on LLRW being stored by generators.

Many generators store LLRW to allow its radioactivity to diminish to levels that permit disposal as non-radioactive waste (i.e., storage for decay). In general, the cognizant regulatory agencies allow storage for decay only where the waste contains radionuclides with half-lives less than 90 days. LLRW in storage for decay is normally held for 10 half-lives, or until radioactivity has diminished to a level where it is indistinguishable from background radiation. Most generators hold LLRW in storage for decay at their own facilities, although approved off-site facilities may be used.

Generators regularly store waste pending transfer to a licensed LLRW disposal facility (i.e., storage pending disposal). Storage pending disposal can occur for extended periods, as when the Barnwell LLRW disposal facility closed to generators in New York from June 30, 1994, until June 30, 1995. Such storage may also occur when the LLRW has a particular characteristic that makes it unacceptable at the available disposal facilities (e.g., contains chemically hazardous components). For those cases where access to licensed disposal facilities is not available, most generators will store LLRW at their own facilities, although approved off-site storage facilities may be used. In addition, most generators routinely store LLRW at their facilities for short periods as a normal part of operation or staging while accumulating a sufficient quantity for transfer to a treatment or disposal facility.

Volume is presented in cubic meters and activity is presented in gigabecquerels (GBq) or megabecquerels (MBq). These units have been adopted to be consistent with NRC uniform national LLRW manifest requirements. The Conversions for Units table on the inside back cover and footnotes to the relevant tables provide information for converting the data to the previously used units of cubic feet and curies.

Individual entries in the following tables have been rounded using standard procedures. Because the totals shown represent the sum of the rounded entries, they may vary slightly from one table to another and may not always equal $100 \%$. Waste volumes have been rounded to the nearest 10 th of a cubic meter. In most cases, activity has been rounded to the nearest 10,000 th of a GBq. Percentages have been rounded to the nearest 10 th of a percent in the tables and figures. 
Table 2-1: Generators Reporting and Storing Waste Pending Disposal ${ }^{1}$

Generator Type

Number Reporting

Number Storing

MEDICAL

Government

1

Private

210

4

College

20

6

Other

$$
5
$$

Total Medical

256

INDUSTRIAL

Manufacturing

10

5

Research \& Development

17

Other

Total Industrial

14

ACADEMIC (non-medical)

College or University

32

12

Other

Total Academic

GOVERNMENT (non-medical)

New York State

2

1

Other

6

Total Government

8

TOTAL

1 Includes LLRW in storage at generator sites or an approved off-site location pending transfer to a licensed LLRW facility, as of December 31, 1998. Does not include LLRW held in storage for decay. 
Table 2-2: Volume and Activity of Waste Stored Pending Disposal ${ }^{1}$

\begin{tabular}{|c|c|c|c|c|}
\hline Generator Type & $\begin{array}{c}\text { Volume } \\
\left(\mathbf{m}^{3}\right)^{2}\end{array}$ & $\begin{array}{l}\% \text { of } \\
\text { Total } \\
\end{array}$ & $\begin{array}{r}\text { Activity } \\
(\mathbf{G B q})^{2}\end{array}$ & $\begin{array}{l}\% \text { of } \\
\text { Total }\end{array}$ \\
\hline \multicolumn{5}{|l|}{ MEDICAL } \\
\hline Government & 0.4 & & 3.7000 & \\
\hline Private & 17.6 & & 2.1995 & \\
\hline College & 25.5 & & 20.0413 & \\
\hline Total Medical & 43.5 & 11.8 & 25.9408 & 0.1 \\
\hline
\end{tabular}

INDUSTRIAL

Manufacturing

11.8

181.9919

Research \& Development

6.2892

Other

0.0009

Total Industrial

15.6

4.2

188.2820

0.6

ACADEMIC (non-medical)

College or University

515.5016

Other

Total Academic

6.6

516.1026

1.6

GOVERNMENT (non-medical)

New York State

45.6

456.9676

Other

0.1295

Total Government

46.5

12.6

457.0971

1.4

TOTAL NON-POWER PLANT

129.9

35.2

$1,187.4225$

3.7

NUCLEAR POWER PLANT

240.0

64.8

$30,522.6816$

96.3

TOTAL

369.9

100.0

31,710.1041

100.0

$\left(13,061 \mathrm{ft}^{3}\right)$

(857 curies)

1 Includes LLRW in storage at generator sites or an approved off-site location pending transfer to a licensed LLRW facility, as of December 31, 1998. Does not include LLRW held in storage for decay.

2 To obtain volume in cubic feet, multiply the number of cubic meters by 35.31 . To obtain activity in curies, divide the number of gigabecquerels $(\mathrm{GBq})$ by 37 
Table 2-3: Waste in Storage Pending Disposal, by Class ${ }^{1}$ and Generator Type ${ }^{2}$

\begin{tabular}{|c|c|c|c|c|c|c|}
\hline \multirow[b]{2}{*}{ Generator Type } & \multicolumn{2}{|c|}{ Class A } & \multicolumn{2}{|c|}{ Class B } & \multicolumn{2}{|c|}{ Class C } \\
\hline & $\begin{array}{c}\text { Volume } \\
\left(\mathbf{m}^{3}\right)^{3}\end{array}$ & $\begin{array}{l}\text { Activity } \\
\text { (GBq) }^{3}\end{array}$ & $\begin{array}{c}\text { Volume } \\
\left(\mathbf{m}^{3}\right)^{3}\end{array}$ & $\begin{array}{l}\text { Activity } \\
(\mathrm{GBq})^{3}\end{array}$ & $\begin{array}{c}\text { Volume } \\
\left(\mathrm{m}^{3}\right)^{3}\end{array}$ & $\begin{array}{r}\text { Activity } \\
(\mathrm{GBq})^{3} \\
\end{array}$ \\
\hline MEDICAL & 43.5 & 25.9109 & 0.0 & 0.0000 & 0.0 & 0.0000 \\
\hline INDUSTRIAL & 15.6 & 188.2820 & 0.0 & 0.0000 & 0.0 & 0.0000 \\
\hline ACADEMIC & 24.0 & 506.7591 & 0.2 & 9.2500 & 0.1 & 0.0185 \\
\hline GOVERNMENT & 46.5 & 457.0971 & 0.0 & 0.0000 & 0.0 & 0.0000 \\
\hline $\begin{array}{l}\text { NUCLEAR POWER } \\
\text { PLANT }\end{array}$ & 234.3 & $29,835.7865$ & 5.7 & 687.0000 & 0.0 & 0.0000 \\
\hline TOTAL & $\begin{array}{c}363.9 \\
\left(12,849 \mathrm{ft}^{3}\right)\end{array}$ & $\begin{array}{l}31,013.8356 \\
\text { (838 curies) }\end{array}$ & $\begin{array}{c}5.9 \\
\left(208 \mathrm{ft}^{3}\right)\end{array}$ & $\begin{array}{r}696.2500 \\
\text { (19 curies) }\end{array}$ & $\begin{array}{c}0.1 \\
\left(3.5 \mathrm{ft}^{3}\right)\end{array}$ & $\begin{array}{r}0.0185 \\
\text { ( * curies) }\end{array}$ \\
\hline
\end{tabular}

1 Classes A, B, and C are waste-classification categories established by the U.S. Nuclear Regulatory Commission in 'Title 10 of the Code of Federal Regulations, Part 61, "Licensing Requirements for Land Disposal of Radioactive Waste," and adopted by the New York State Department of Environmental Conservation in 6 NYCRR Part 382, "Regulations for Low-Level Radioactive Waste Disposal Facilities."

2 Refers to LLRW in storage at generator sites or an approved off-site location pending transfer to a licensed LLRW facility, as of December 31, 1998. Does not include LLRW held in storage for decay.

${ }^{3}$ To obtain volume in cubic feet, multiply the number of cubic meters by 35.31 . To obtain activity in curies, divide the number of gigabecquerels $(\mathrm{GBq})$ by 37 .

* Less than 0.1 curies. 
Table 2-4: Number of Facilities Reporting Various Waste Types in Storage Pending Disposal

\begin{tabular}{|c|c|c|c|c|c|c|}
\hline Waste Type ${ }^{1}$ & Medical & Industrial & Academic & Government & $\begin{array}{c}\text { Nuclear } \\
\text { Power } \\
\text { Plants } \\
\end{array}$ & Total \\
\hline Aqueous Liquids & 4 & 4 & 3 & 0 & 0 & 11 \\
\hline $\begin{array}{l}\text { Biological Material (excluding } \\
\text { carcasses) }\end{array}$ & 0 & 0 & 1 & 0 & 0 & 1 \\
\hline Charcoal & 0 & 1 & 0 & 0 & 2 & 3 \\
\hline Compacted Trash & 4 & 2 & 5 & 0 & 0 & 11 \\
\hline Glassware/Labware & 1 & 0 & 1 & 0 & 0 & 2 \\
\hline Ion Exchange Media & 0 & 0 & 0 & 0 & 1 & 1 \\
\hline Mechanical Filter & 0 & 1 & 0 & 0 & 1 & 2 \\
\hline Non-compacted Trash & 0 & 1 & 0 & 0 & 0 & 1 \\
\hline $\begin{array}{l}\text { Organic Liquids (excluding } \\
\text { oil) }\end{array}$ & 0 & 0 & 1 & 0 & 0 & 1 \\
\hline Sealed Source/Device & 2 & 4 & 1 & 0 & 0 & 7 \\
\hline Soil & 0 & 1 & 1 & 1 & 0 & 3 \\
\hline Other ${ }^{2}$ & 0 & 0 & 0 & 1 & 0 & 1 \\
\hline
\end{tabular}

Unless otherwise noted, all data were derived from low-level radioactive waste generator reports received by NYSERDA as of May 4, 1999. 
Table 2-5: Waste in Storage ${ }^{1}$ Pending Disposal, by County of Origin ${ }^{2}$

\begin{tabular}{|c|c|c|c|c|c|c|}
\hline County & $\begin{array}{l}\text { Number of } \\
\text { Generators } \\
\text { Reporting }\end{array}$ & $\begin{array}{c}\text { Number of } \\
\text { Generators } \\
\text { Storing LLRW } \\
\end{array}$ & $\begin{array}{c}\text { Volume } \\
\left(\mathrm{m}^{3}\right)^{4}\end{array}$ & $\begin{array}{l}\% \text { of } \\
\text { Total }\end{array}$ & $\begin{array}{l}\text { Activity } \\
\text { (GBq) }^{4}\end{array}$ & $\%$ of Total \\
\hline Albany & 11 & 2 & 1.1 & 0.3 & 0.5245 & $*$ \\
\hline Allegany & 0 & 0 & 0.0 & 0.0 & 0.0000 & 0.0 \\
\hline Bronx & 12 & 1 & 0.2 & $*$ & 0.7400 & 0.0 \\
\hline Broome & 6 & 2 & 0.3 & 0.1 & 5.0794 & $*$ \\
\hline Cattaraugus & 1 & 1 & 45.6 & 12.3 & 456.9676 & 1.4 \\
\hline Cayuga & 1 & 0 & 0.0 & 0.0 & 0.0000 & 0.0 \\
\hline Chautauqua & 2 & 0 & 0.0 & 0.0 & 0.0000 & 0.0 \\
\hline Chemung & 4 & 1 & 0.2 & $*$ & 0.0037 & * \\
\hline Chenango & 0 & 0 & 0.0 & 0.0 & 0.0000 & 0.0 \\
\hline Clinton & 4 & 1 & $*$ & $*$ & 0.9262 & $*$ \\
\hline Columbia & 0 & 0 & 0.0 & 0.0 & 0.0000 & 0.0 \\
\hline Cortland & 1 & 0 & 0.0 & 0.0 & 0.0000 & 0.0 \\
\hline Delaware & 2 & 0 & 0.0 & 0.0 & 0.0000 & 0.0 \\
\hline Dutchess & 8 & 1 & 2.1 & 0.6 & 1.8500 & * \\
\hline Erie & 20 & 3 & 5.1 & 1.4 & 0.1886 & $*$ \\
\hline Essex & 3 & 1 & 0.2 & $*$ & 0.1180 & $*$ \\
\hline Franklin & 2 & 0 & 0.0 & 0.0 & 0.0000 & 0.0 \\
\hline Fulton & 0 & 0 & 0.0 & 0.0 & 0.0000 & 0.0 \\
\hline Genesee & 1 & 0 & 0.0 & 0.0 & 0.0000 & 0.0 \\
\hline Greene & 0 & 0 & 0.0 & 0.0 & 0.0000 & 0.0 \\
\hline Hamilton & 0 & 0 & 0.0 & 0.0 & 0.0000 & 0.0 \\
\hline Herkimer & 1 & 0 & 0.0 & 0.0 & 0.0000 & 0.0 \\
\hline Jefferson & 3 & 0 & 0.0 & 0.0 & 0.0000 & 0.0 \\
\hline Kings & 18 & 0 & 0.0 & 0.0 & 0.0000 & 0.0 \\
\hline Lewis & 0 & 0 & 0.0 & 0.0 & 0.0000 & 0.0 \\
\hline Livingston & 1 & 0 & 0.0 & 0.0 & 0.0000 & 0.0 \\
\hline Madison & 2 & 0 & 0.0 & 0.0 & 0.0000 & 0.0 \\
\hline Monroe & 12 & 2 & 5.4 & 1.5 & 5.3649 & * \\
\hline Montgomery & 2 & 0 & 0.0 & 0.0 & 0.0000 & 0.0 \\
\hline Nassau & 34 & 4 & 2.3 & 0.6 & 1.6472 & $*$ \\
\hline New York & 32 & 7 & 34.8 & 9.4 & 515.8859 & 1.6 \\
\hline Niagara & 2 & 0 & 0.0 & 0.0 & 0.0000 & 0.0 \\
\hline Oneida & 4 & 1 & 0.8 & 0.2 & 9.4123 & * \\
\hline Onondaga & 11 & 0 & 0.0 & 0.0 & 0.0000 & 0.0 \\
\hline
\end{tabular}

Unless otherwise noted, all data were derived from low-level radioactive waste generator reports received by NYSERDA as of May 4, 1999.

New York State Low-Level Radioactive Waste Status Report for 1998 
Table 2-5: Waste in Storage ${ }^{1}$ Pending Disposal, by County of Origin $^{2}$ (continued)

\begin{tabular}{|c|c|c|c|c|c|c|}
\hline County & $\begin{array}{l}\text { Number of } \\
\text { Generators } \\
\text { Reporting }\end{array}$ & $\begin{array}{c}\text { Number of } \\
\text { Generators } \\
\text { Storing LLRW } \\
\end{array}$ & $\begin{array}{c}\text { Volume } \\
\left(\mathrm{m}^{3}\right)^{4}\end{array}$ & $\begin{array}{l}\% \text { of } \\
\text { Total }\end{array}$ & $\begin{array}{l}\text { Activity } \\
\text { (GBq) }^{4}\end{array}$ & $\%$ of Total \\
\hline Ontario & 3 & 0 & 0.0 & 0.0 & 0.0000 & 0.0 \\
\hline Orange & 8 & 0 & 0.0 & 0.0 & 0.0000 & 0.0 \\
\hline Orleans & 1 & 0 & 0.0 & 0.0 & 0.0000 & 0.0 \\
\hline Oswego & 3 & 3 & 235.6 & 63.7 & $30,520.9516$ & 96.3 \\
\hline Otsego & 3 & 0 & 0.0 & 0.0 & 0.0000 & 0.0 \\
\hline Putnam & 2 & 1 & 0.3 & 0.1 & 0.1191 & $*$ \\
\hline Queens & 13 & 1 & 0.8 & 0.2 & 0.3090 & $*$ \\
\hline Rensselaer & 4 & 3 & 16.2 & 4.4 & 4.3207 & $*$ \\
\hline Richmond & 5 & 1 & 0.4 & 0.1 & 0.0600 & $*$ \\
\hline Rockland & 8 & 2 & 4.0 & 1.1 & 176.9743 & 0.6 \\
\hline St. Lawrence & 3 & 1 & 0.1 & $*$ & 0.2080 & * \\
\hline Saratoga & 3 & 0 & 0.0 & 0.0 & 0.0000 & 0.0 \\
\hline Schenectady & 4 & 1 & 0.2 & $*$ & 2.3852 & * \\
\hline Schoharie & 0 & 0 & 0.0 & 0.0 & 0.0000 & 0.0 \\
\hline Schuyler & 0 & 0 & 0.0 & 0.0 & 0.0000 & 0.0 \\
\hline Seneca & 1 & 0 & 0.0 & 0.0 & 0.0000 & 0.0 \\
\hline Steuben & 2 & 0 & 0.0 & 0.0 & 0.0000 & 0.0 \\
\hline Suffolk & 33 & 1 & 1.7 & 0.5 & 2.0000 & $*$ \\
\hline Sullivan & 0 & 0 & 0.0 & 0.0 & 0.0000 & 0.0 \\
\hline Tioga & 0 & 0 & 0.0 & 0.0 & 0.0000 & 0.0 \\
\hline Tompkins & 3 & 1 & 0.3 & 0.1 & 0.3288 & $*$ \\
\hline Ulster & 6 & 0 & 0.0 & 0.0 & 0.0000 & 0.0 \\
\hline Warren & 1 & 0 & 0.0 & 0.0 & 0.0000 & 0.0 \\
\hline Washington & 0 & 0 & 0.0 & 0.0 & 0.0000 & 0.0 \\
\hline Wayne & 2 & 1 & 4.4 & 1.2 & 1.7300 & $*$ \\
\hline Westchester & 26 & 2 & 7.8 & 2.1 & 2.0091 & $*$ \\
\hline Wyoming & 0 & 0 & 0.0 & 0.0 & 0.0000 & 0.0 \\
\hline Yates & 0 & 0 & 0.0 & 0.0 & 0.0000 & 0.0 \\
\hline TOTALS & 334 & 45 & $\begin{array}{r}369.9 \\
\left(13,061 \mathrm{ft}^{3}\right)\end{array}$ & 99.9 & $\begin{array}{l}31,710.1041 \\
(857 \text { curies) }\end{array}$ & 99.9 \\
\hline \multicolumn{7}{|c|}{$\begin{array}{l}\text { Includes LLRW in storage at generator sites or an approved off-site location pending transfer to a licensed LLRW facility, as of } \\
\text { December } 31,1998 \text {. Does not include LLRW held in storage for decay. } \\
\text { Section } 4 \text { of this report identifies the individual facilities reporting LLRW in storage pending disposal. } \\
\text { Refers to the number of generators who reported LLRW in storage pending disposal as of December } 31,1998 \text {. } \\
\text { To obtain volume in cubic feet, multiply the number of cubic meters by } 35.31 \text {. To obtain activity in curies, divide the number of } \\
\text { gigabecquerels (GBq) by } 37 \text {. } \\
\text { Less than } 0.1 \text { cubic meter or } 0.1 \% \text {. }\end{array}$} \\
\hline
\end{tabular}

Unless otherwise noted, all data were derived from low-level radioactive waste generator reports received by NYSERDA as of May 4, 1999.

New York State Low-Level Radioactive Waste Status Report for 1998

New York State Energy Research and Development Authority - June 1999 
Table 2-6: Radionuclide Content ${ }^{1}$ of Waste in Storage Pending Disposal (in MBq) ${ }^{2}$

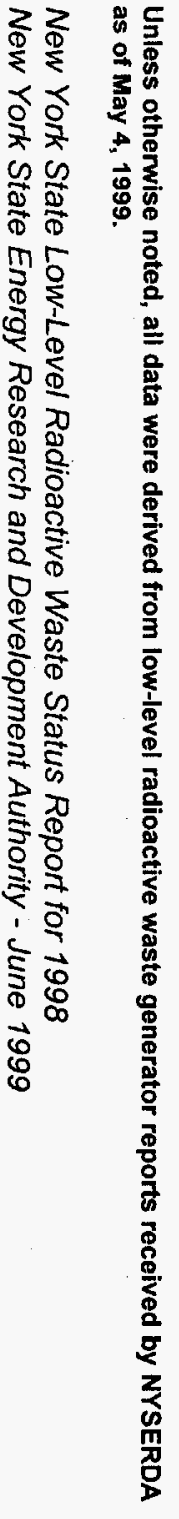

\begin{tabular}{|c|c|c|c|c|c|c|c|}
\hline Radionuclide & Half-Life ${ }^{3,4}$ & Academic & Government & Industrial & Medical & $\begin{array}{c}\text { Nuclear Power } \\
\text { Plants } \\
\end{array}$ & Total \\
\hline $\mathrm{Ag}-110 \mathrm{~m}$ & $21.8 \mathrm{y}$ & & & & & $18,224.220$ & $18,224.220$ \\
\hline $\mathrm{Al}-26$ & 7.1 E5 y & & & & 18.520 & & 18.520 \\
\hline Am-241 & $432.7 y$ & & $*$ & $3,515.000$ & $4,000.000$ & & $7,515.000$ \\
\hline$A m-242$ & $1141 \mathrm{y}$ & & & & & 50.890 & 50.890 \\
\hline Ba-133 & $10.5 \mathrm{y}$ & 0.111 & & & 0.037 & & 0.148 \\
\hline$C-14$ & $5730 y$ & 651.500 & $7,510.000$ & $1,251.440$ & $5,876.610$ & $56,670.050$ & $71,959.600$ \\
\hline $\mathrm{Ca}-45$ & $162.7 \mathrm{~d}$ & 0.002 & & & 18.520 & & 18.522 \\
\hline Cd-109 & $462.0 \mathrm{~d}$ & & $*$ & & & & * \\
\hline $\mathrm{Ce}-141$ & $32.5 \mathrm{~d}$ & & & & & 0.343 & 0.343 \\
\hline $\mathrm{Ce}-144$ & $284.6 \mathrm{~d}$ & & & & & $73,579.920$ & $73,579.920$ \\
\hline $\mathrm{Cf}-252$ & $2.65 y$ & & & 0.111 & 0.054 & & 0.165 \\
\hline $\mathrm{Cm}-242$ & $162.8 \mathrm{~d}$ & & & & & 12.310 & 12.310 \\
\hline $\mathrm{Cm}-243$ & $29.1 \mathrm{y}$ & & & & & 34.710 & 34.710 \\
\hline $\mathrm{Cm}-243 / 244$ & $29.1 \mathrm{y}$ & & & & & 0.171 & 0.171 \\
\hline $\mathrm{Cm}-244$ & $18.1 \mathrm{y}$ & & & & 25.890 & & 25.890 \\
\hline $\mathrm{Co}-57$ & $271.8 \mathrm{~d}$ & & $*$ & 158.920 & & 196.500 & 355.420 \\
\hline $\mathrm{Co}-58$ & $70.9 \mathrm{~d}$ & & & 0.250 & & $12,904.930$ & $12,905.180$ \\
\hline Co-60 & $5.2 \mathrm{y}$ & $1,098.043$ & $*$ & 4.098 & & $9,974,496.983$ & $9,975,599.124$ \\
\hline Cr-51 & $27.7 \mathrm{~d}$ & & $*$ & 481.250 & 410.700 & $22,528.102$ & $23,420.052$ \\
\hline Cs-134 & $2.0 \mathrm{y}$ & & & & & $40,100.000$ & $40,100.000$ \\
\hline Cs-137 & $30.2 y$ & $500,000.576$ & 252.000 & 0.370 & $*$ & $807,563.863$ & $1,307,816.809$ \\
\hline $\mathrm{Fe}-55$ & $2.7 \mathrm{y}$ & & & & & $2,697,497.890$ & $2,697,497.890$ \\
\hline $\mathrm{Fe}-59$ & $44.5 \mathrm{~d}$ & & & & & $10,033.710$ & $10,033.710$ \\
\hline Gd-153 & $241.6 \mathrm{~d}$ & 0.037 & & & 18.520 & & 18.557 \\
\hline $\mathrm{H}-3$ & $12.3 y$ & $14,335.620$ & $449,000.000$ & $179,941.420$ & $9,689.830$ & $9,553.210$ & $662,520.080$ \\
\hline $\mathrm{I}-125$ & $59.4 \mathrm{~d}$ & & & $\begin{array}{r}84.370 \\
\end{array}$ & 395.900 & & 480.270 \\
\hline $\mathrm{I}-129$ & $1.6 \mathrm{E} 7 \mathrm{y}$ & 0.004 & & & & 20.830 & 20.834 \\
\hline $\mathrm{Kr}-85$ & $10.8 \mathrm{y}$ & & & 370.000 & & & 370.000 \\
\hline $\mathrm{Mn}-54$ & $312.2 \mathrm{~d}$ & 0.074 & & 0.250 & & $977,764.220$ & $977,764.544$ \\
\hline $\mathrm{Na}-22$ & $2.6 \mathrm{y}$ & 0.388 & & & & & 0.388 \\
\hline $\mathrm{Nb}-94$ & $2.0 \mathrm{E} 4 \mathrm{y}$ & & & & 18.520 & $2,708.000$ & $2,726.520$ \\
\hline
\end{tabular}




\begin{tabular}{|c|c|c|c|c|c|c|c|c|}
\hline \multirow{26}{*}{ 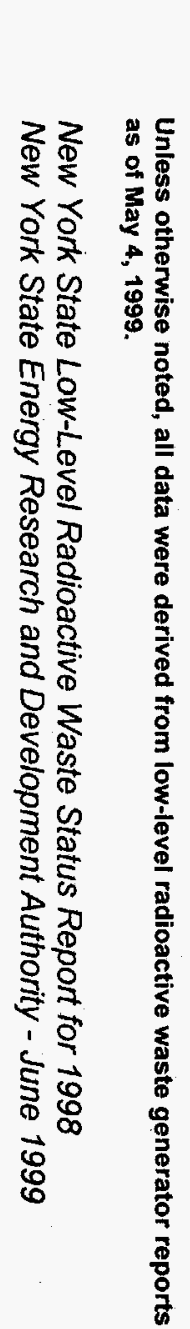 } & $\mathrm{Nb}-95$ & $35.0 \mathrm{~d}$ & & & & & 12.800 & 12.800 \\
\hline & $\mathrm{Ni}-59$ & $7.6 \mathrm{E} 4 \mathrm{y}$ & & & & & $60,766.900$ & $60,766.900$ \\
\hline & $\mathrm{Ni}-63$ & $100 \mathrm{y}$ & 0.190 & & 481.000 & & $524,528.320$ & $525,009.510$ \\
\hline & P-32 & $14.3 \mathrm{~d}$ & & & 22.220 & 477.300 & & 499.520 \\
\hline & $\mathrm{P}-33$ & $25.3 \mathrm{~d}$ & & & 752.950 & & & 752.950 \\
\hline & $\mathrm{Pb}-210$ & $22.6 \mathrm{y}$ & 0.087 & & 0.004 & 0.186 & & 0.277 \\
\hline & Po-210 & $138.4 \mathrm{~d}$ & & $*$ & & & & * \\
\hline & $\mathrm{Pu}-238$ & $87.7 y$ & & & $1,110.000$ & & 29.760 & $1,139.760$ \\
\hline & $\mathrm{Pu}-239$ & $2.4 \mathrm{E} 4 \mathrm{y}$ & & & & & 18.270 & 18.270 \\
\hline & $\mathrm{Pu}-241$ & $14.4 \mathrm{y}$ & & & & & $19,760.294$ & $19,760.294$ \\
\hline & Ra-226 & $1.6 \mathrm{E} 3 \mathrm{y}$ & 0.070 & & 0.796 & 112.000 & & 112.866 \\
\hline & $S-35$ & $87.2 \mathrm{~d}$ & & & 88.800 & 244.200 & & 333.000 \\
\hline & $\mathrm{Sb}-125$ & $2.8 \mathrm{y}$ & & & & & 77.850 & 77.850 \\
\hline & $\mathrm{Sm}-121$ & $1.1 \mathrm{~d}$ & & & & 18.520 & & 18.520 \\
\hline & Sn-113 & $115.1 \mathrm{~d}$ & & * & $*$ & 37.040 & & 37.040 \\
\hline & Sr-85 & $64.8 \mathrm{~d}$ & & & & 18.520 & & 18.520 \\
\hline & $\mathrm{Sr}-89$ & $50.5 \mathrm{~d}$ & & & & & 38.080 & 38.080 \\
\hline & Sr-90 & $29.1 \mathrm{y}$ & 0.052 & 107.000 & & $4,597.000$ & $3,284.393$ & $7,988.445$ \\
\hline & Tc-99 & $2.1 \mathrm{E} 5 \mathrm{y}$ & & 98.600 & & & $4,293.700$ & $4,392.300$ \\
\hline & Tc-99m & $6.0 \mathrm{~h}$ & & & 18.500 & & & 18.500 \\
\hline & Th-232 & $1.4 \mathrm{E} 10 \mathrm{y}$ & 0.037 & & & & & 0.037 \\
\hline & Tl-204 & $3.8 y$ & 0.037 & & & & & 0.037 \\
\hline & U-238 & $4.5 \mathrm{E} 9 \mathrm{y}$ & 13.680 & 129.500 & & 0.001 & & 143.181 \\
\hline & $\mathrm{Zn}-65$ & $243.8 \mathrm{~d}$ & & & 0.256 & & $15,205,828.000$ & $15,205,828.256$ \\
\hline & Zr-95 & $64 \mathrm{~d}$ & & & & & $\quad 67.470$ & 67.470 \\
\hline & Total & & $516,100.508$ & $457,097.100$ & 188.282 .005 & $25,977.868$ & $30,522,646.689$ & $31,710,104.170$ \\
\hline
\end{tabular}

' Some generator facilities have reported radionuclides with half-lives of less than 90 days in LLRW held for storage pending disposal. In the majority of these cases, the shorter-lived radionuclides reported cannot be separated readily from longer-lived radionuclides in the waste. Does not include LLRW in storage for decay.

${ }^{2}$ To obtain activity in curies, divide the number of $\mathrm{MBq}$ by 37,000 .

${ }^{3}$ Source: Chart of the Nuclides, General Electric Company under the direction of Naval Reactors, U.S. DOE; 15th edition, revised to 1996. NB: $y=y e a r s, d=d a y s, h=h o u r s$

${ }^{4}$ Where scientific notation is used, multiply the number by 10 to the specified power. Example: For Ra-226, $1.6 \mathrm{E} 3=1.6 \times 10^{3}=1.6 \times 10 \times 10 \times 10=1.6 \times 1,000=1,600$ For this table, the whole number to which 10 is raised is equal to the number of places the decimal is moved to the right. 


\begin{tabular}{|c|c|c|c|c|}
\hline Generator Type & $\begin{array}{l}\text { Number of } \\
\text { Generators } \\
\text { Reporting } \\
\end{array}$ & $\begin{array}{l}\text { Number of } \\
\text { Generators } \\
\text { Reporting } \\
\text { Storage for } \\
\text { Decay }^{2} \\
\end{array}$ & $\begin{array}{c}\text { Number of } \\
\text { Generators } \\
\text { Reporting Only } \\
\text { Storage for } \\
\text { Decay } \\
\end{array}$ & $\begin{array}{c}\text { Estimated } \\
\text { Maximum Volume } \\
\text { in Storage for } \\
\text { Decay at Any } \\
\text { Time }\left(\mathrm{m}^{3}\right)^{3} \\
\end{array}$ \\
\hline MEDICAL & 256 & 249 & 220 & 1,286 \\
\hline INDUSTRIAL & 29 & 13 & 7 & 170 \\
\hline ACADEMIC & 35 & 33 & 17 & 359 \\
\hline GOVERNMENT & 8 & 3 & 2 & 51 \\
\hline NUCLEAR POWER PLANT & 6 & 0 & 0 & 0 \\
\hline TOTAL & 334 & 298 & 246 & 1,866 \\
\hline
\end{tabular}

\footnotetext{
Storage for decay means holding the LLRW until the level of radioactivity has diminished to the point where it can be disposed of as non-radioactive waste. Normally, such LLRW is held for 10 half-lives, or until the radioactivity has diminished to a level that is undetectable above background radiation.

Typical radionuclides held for decay, with their respective half-lives, include: Iodine-123 (13.1 hours), Iodine-125 (59.7 days), Iodine-131 (8.04 days), Technetium-99m (6.02 hours), Phosphorous-32 (14.3 days), Gallium-67 (3.26 days), and Sulfur-35 (89.9 days).

2 Some generators who store for decay also may have transferred other LLRW to one of the licensed LLRW disposal facilities or may be storing LLRW pending disposal

3 To obtain volume in cubic feet, multiply the number of cubic meters by 35.31 .
} as of May 4, 1999. 
Table 2-8: Treatments ${ }^{1}$ Reported for Waste in Storage Pending Disposal, by Generator Type

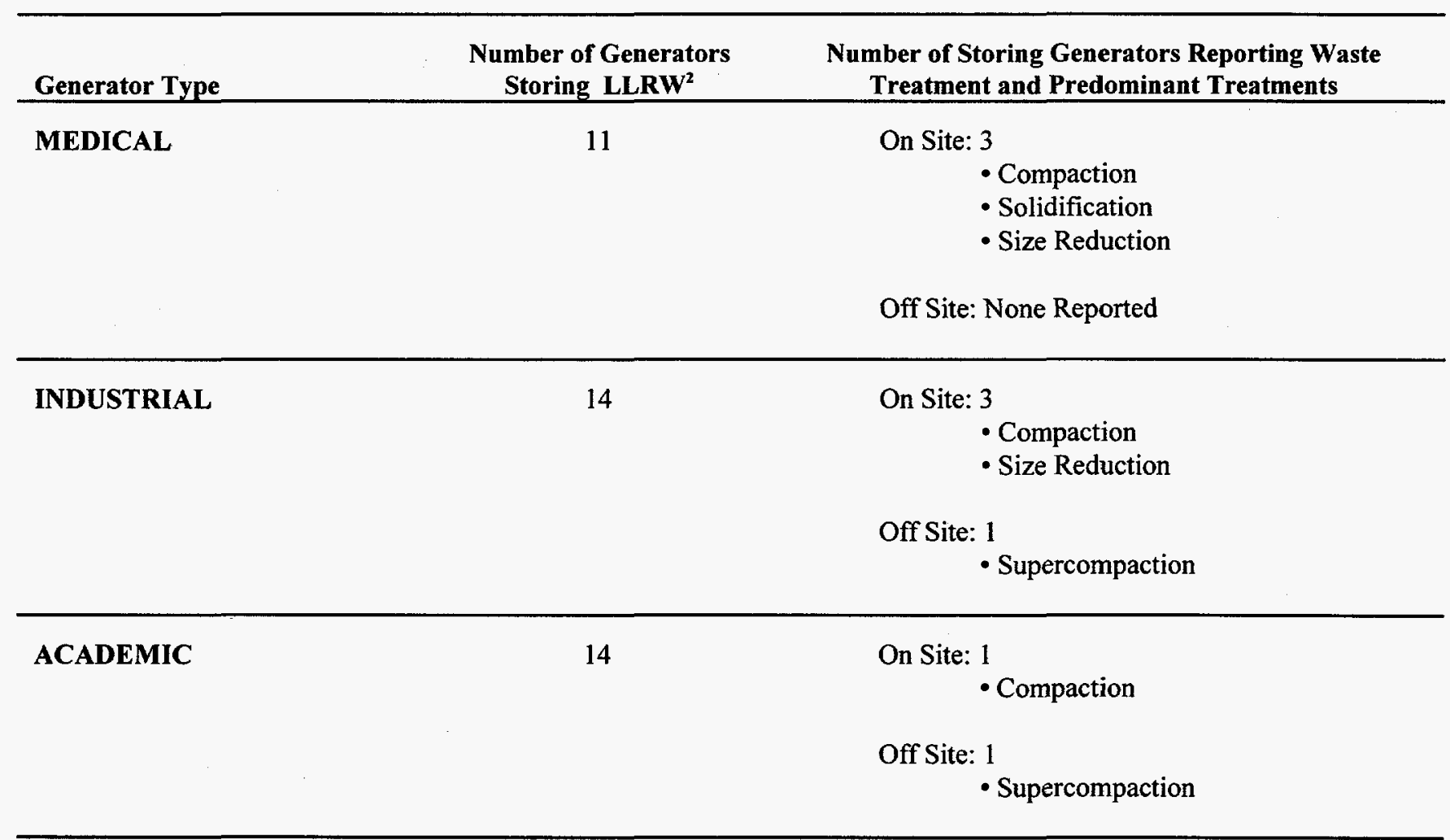

On Site: None Reported

Off Site: None Reported

On Site: None Reported

Off Site: None Reported

1 Treatment refers to processing LLRW to reduce its volume or activity, or change its chemical or physical form, prior to transfer to a disposal facility. Some generators reported using both on-site and off-site waste treatment facilities.

2 Refers to the number of generators who reported storing LLRW pending disposal at their facility or an approved off-site location as of December 31, 1998. 


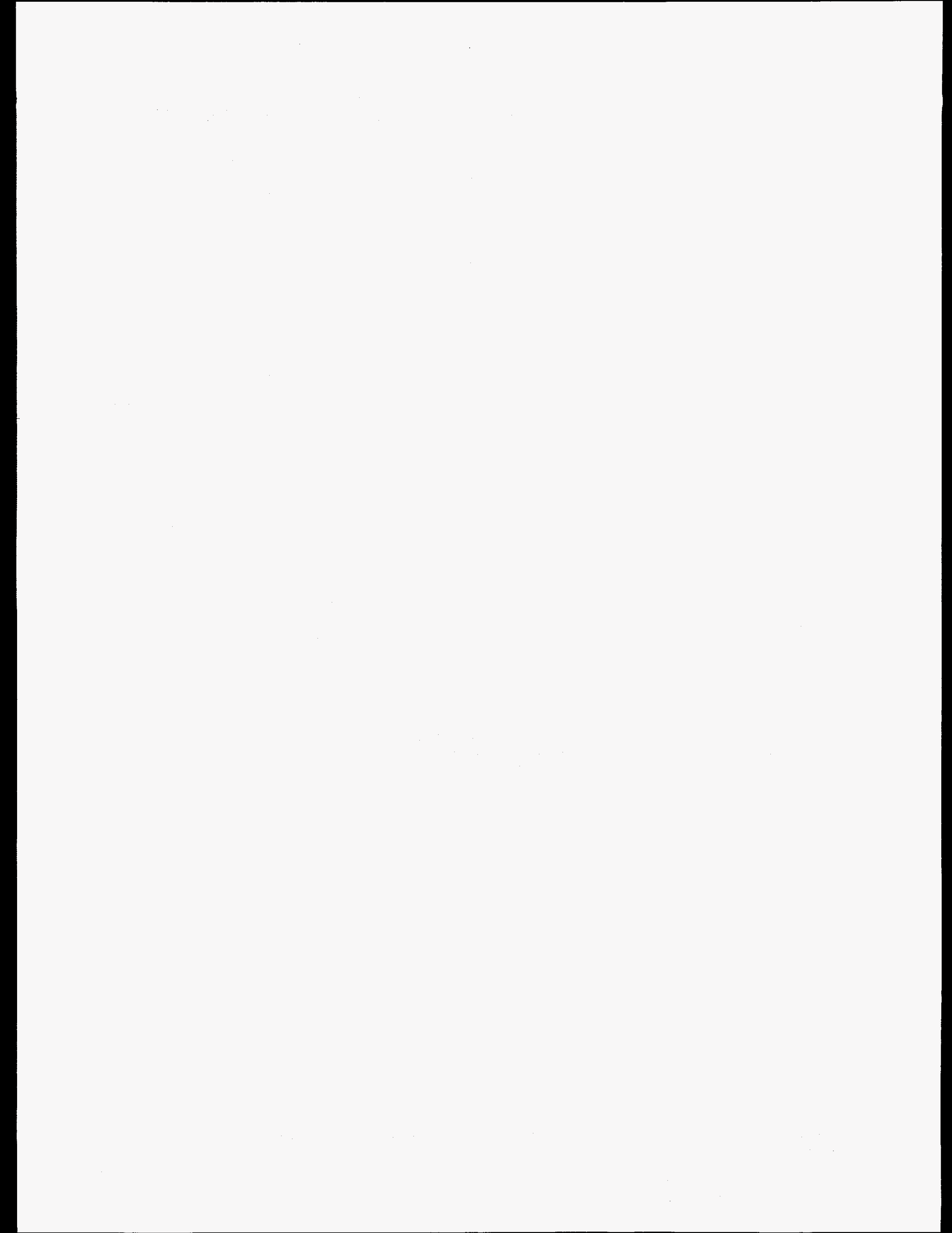


Section 3

\section{HISTORIC DATA AND PROJECTIONS FOR LOW-LEVEL RADIOACTIVE WASTE GENERATION IN NEW YORK STATE}

This section provides historic data on volume and activity of LLRW shipped for disposal, based on generator data reported to NYSERDA for calendar years 1990 through 1998.

This section also provides a summary, based on information supplied in the 1998 generator reports, of generator projections of the volume and activity of LLRW that will require disposal in a licensed LLRW facility for the years 1999 to 2003.

Volume is presented in cubic meters and activity is presented in GBq. These units have been adopted to be consistent with NRC uniform national LLRW manifest requirements. The Conversions for Units table on the inside back cover and footnotes to the relevant tables provide information for converting the data to the previously used units of cubic feet and curies. Volume projections have been rounded to the nearest 10 th of a cubic meter, and activity projections to the nearest $G B q$. 


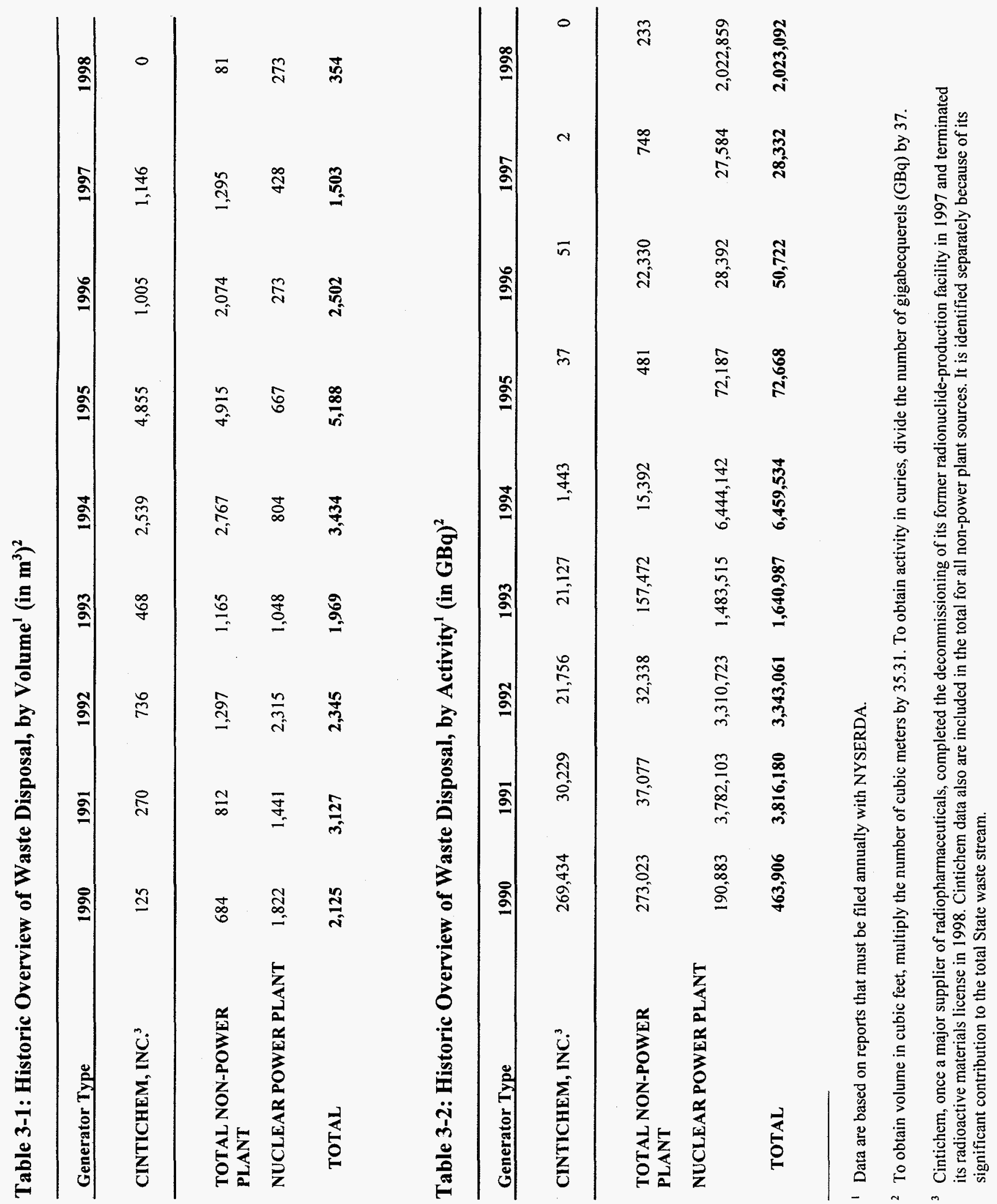



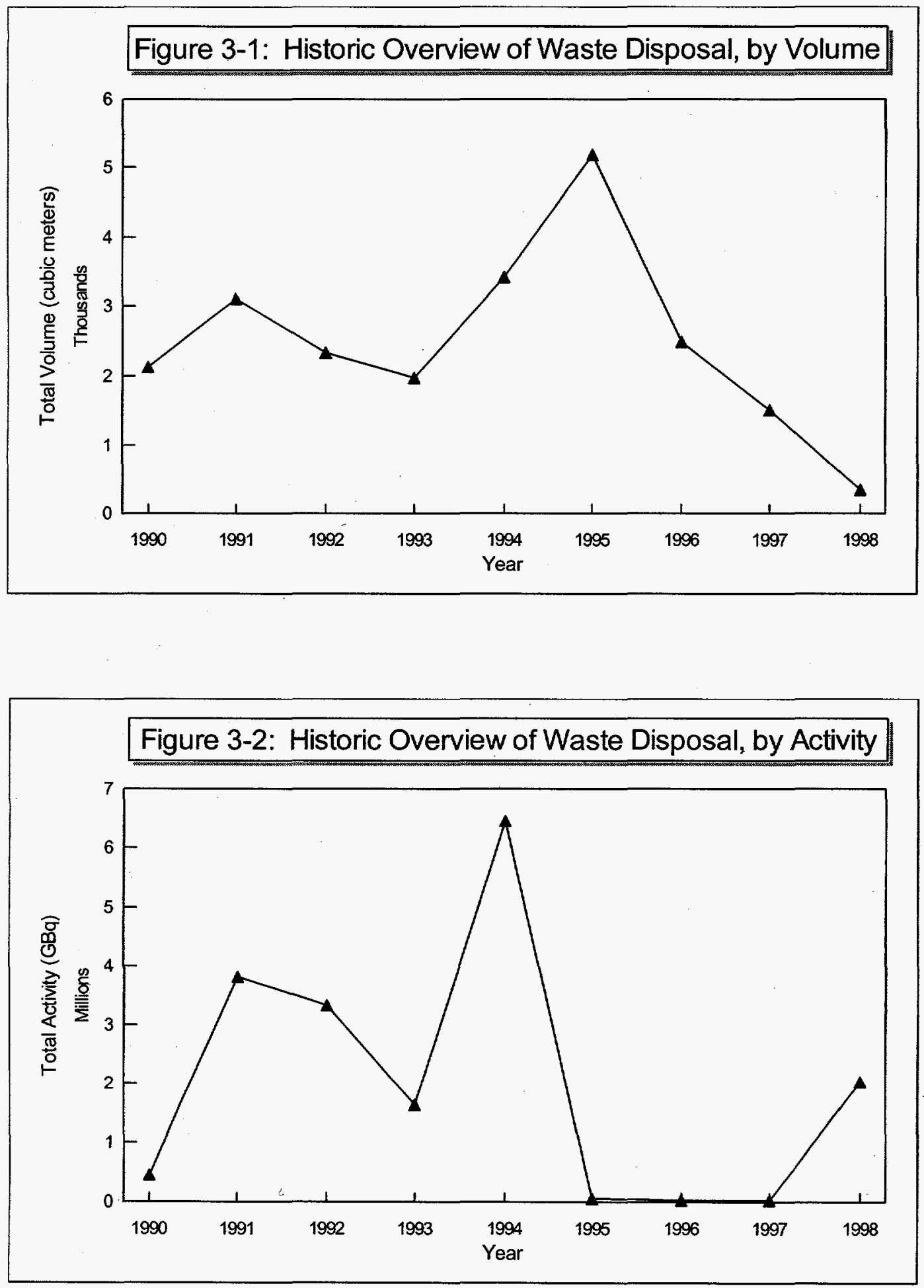

Unless otherwise noted, all data were derived from low-level radioactive waste generator reports received by NYSERDA as of May 4, 1999. 


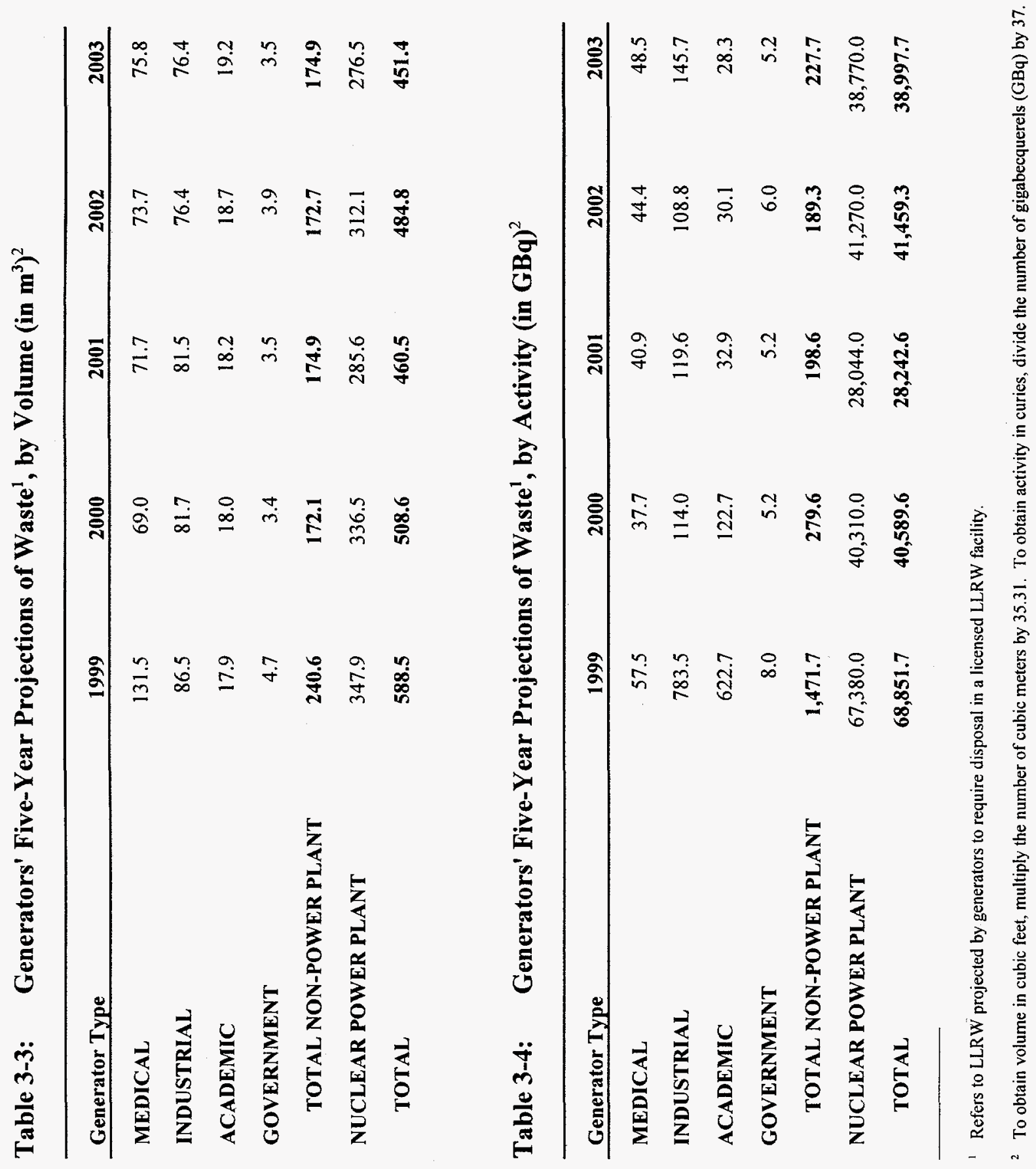

Unless otherwise noted, all data were derived from low-level radioactive waste generator reports received by NYSERDA as of May $4,1999$. 


\section{Section 4}

\section{GENERATORS FILING REPORTS}

This section identifies those facilities that filed LLRW reports with NYSERDA for calendar year 1998 in accordance with the New York State LLRW Management Act (Ch. 673, L. 1986) and NYSERDA regulations (21 NYCRR Part 502).

Table 4a lists the total volume and activity of LLRW reported by generators as having been shipped to LLRW disposal facilities in 1998 and the total volume and activity of LLRW reported by each generator as being held in storage pending disposal as of December 31, 1998.

Table $4 \mathrm{~b}$ lists the generators reporting LLRW held only in storage for decay during 1998.

Generator estimates of total storage capacity and the time that LLRW can continue to be produced and stored on site, absent access to disposal facilities, also are included. The table indicates where storage capacity includes both storage pending transfer to a LLRW disposal facility and storage for decay. The absence of data indicates that the generator reported no information in the particular category.

Volume is presented in cubic meters and activity is presented in gigabecquerels (GBq). These units have been adopted to be consistent with new NRC uniform national LLRW manifest requirements. The Conversions for Units table on the inside back cover and footnotes to the relevant tables provide information for converting the data to the previously used units of cubic feet and curies.

The individual entries in this section have been rounded using standard procedures. Waste volumes have been rounded to the nearest 10 th of a cubic meter. Activity has been rounded to the nearest 10,000th of a gigabecquerel. An asterisk $\left({ }^{*}\right)$ indicates an activity of less than a 10,000 th of a GBq or a volume of less than a 10 th of a cubic meter. 


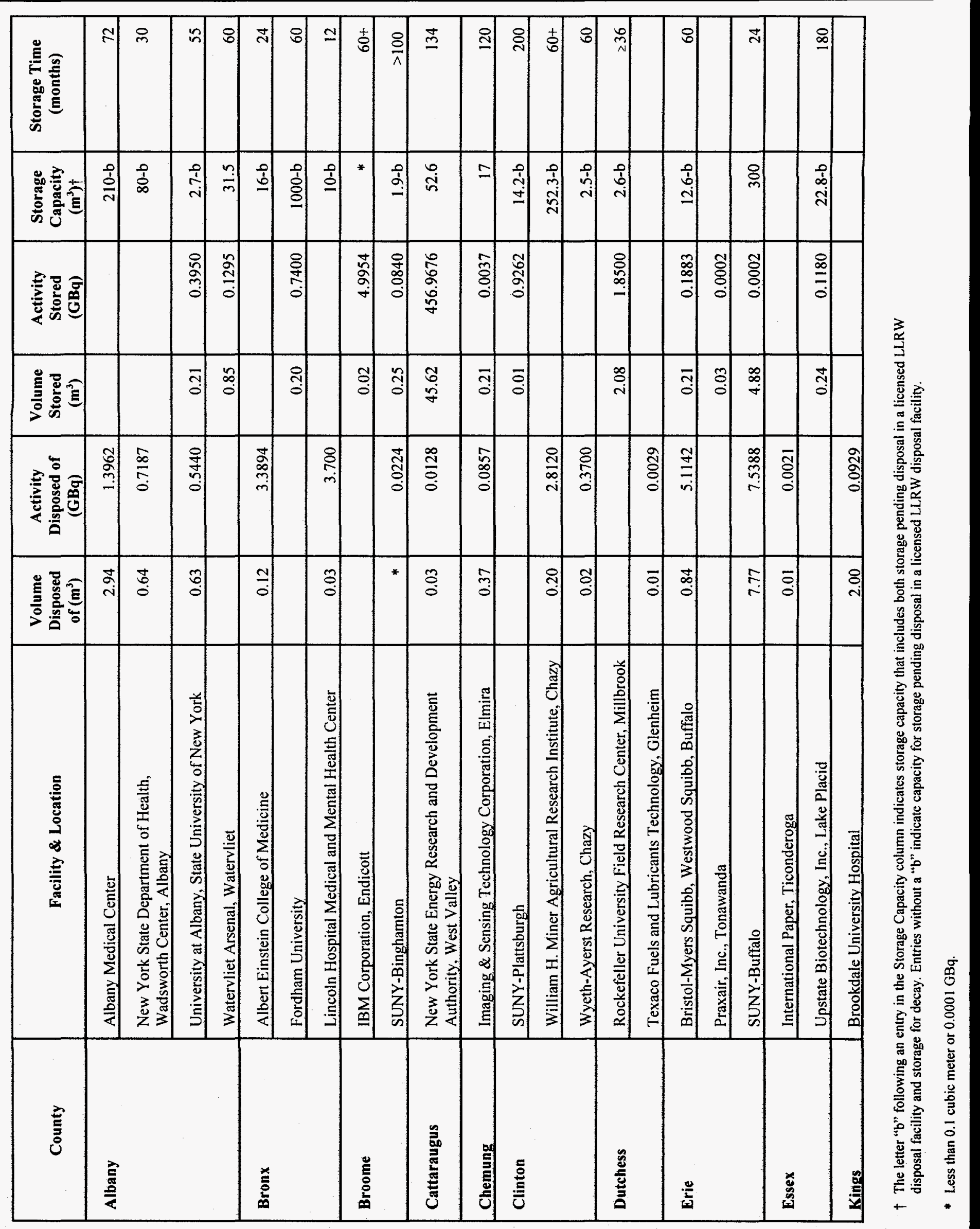




\begin{tabular}{|c|c|c|c|c|c|c|c|c|}
\hline \multirow{20}{*}{ 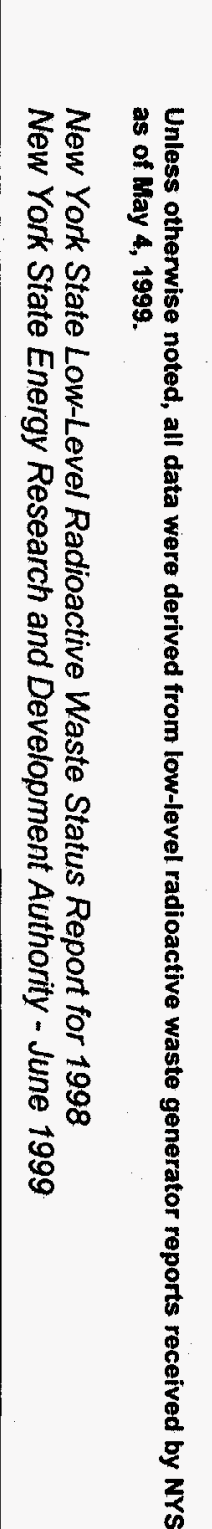 } & County & Facility \& Location & $\begin{array}{c}\begin{array}{c}\text { Volume } \\
\text { Disposed } \\
\text { of }\left(\mathbf{m}^{3}\right)\end{array} \\
\end{array}$ & $\begin{array}{c}\text { Activity } \\
\text { Disposed of } \\
\text { (GBq) } \\
\end{array}$ & $\begin{array}{c}\text { Volume } \\
\text { Stored } \\
\left(\mathrm{m}^{3}\right) \\
\end{array}$ & $\begin{array}{l}\text { Activity } \\
\text { Stored } \\
(\text { GBq) } \\
\end{array}$ & $\begin{array}{c}\begin{array}{c}\text { Storage } \\
\text { Capacity } \\
\left(\mathrm{m}^{3}\right) \dagger\end{array} \\
\end{array}$ & $\begin{array}{l}\text { Storage Time } \\
\text { (months) }\end{array}$ \\
\hline & Kings & New York Harbor Health Care System & 0.53 & 0.0130 & & & $249-b$ & 69 \\
\hline & Monroe & Astra-Arcus USA, Rochester & 0.16 & 0.4476 & & & 146.4-b & 60 \\
\hline & & Eastman Kodak Company' & & & & & & \\
\hline & & SUNY College at Brockport & & & 0.42 & 0.1480 & $0.8-b$ & 24 \\
\hline & Nassau & Cold Spring Harbor Laboratory & 0.56 & 0.1110 & 0.56 & 0.1110 & $90-b$ & 120 \\
\hline & & North Shore University Hospital, Manhasset & 0.54 & 0.5772 & & & $18.2-b$ & 36 \\
\hline & & Northrup Grumman Corporation, Bethpage & & & 0.04 & 0.4811 & 560 & 12 \\
\hline & & $\begin{array}{l}\text { New York College of Osteopathic Medicine of NYIT, } \\
\text { Old Westbury }\end{array}$ & & & 0.04 & 0.0326 & $1.3-b$ & 60 \\
\hline & & OSI Pharmaceuticals, Inc., Uniondale & 1.68 & 1.4040 & 1.68 & 1.0225 & $3.9 \cdot b$ & 12 \\
\hline & & Winthrop University Hospital, Mineola & 2.20 & 0.1113 & & & 179-b & 120 \\
\hline & New York & Bell Atlantic ${ }^{2}$ & & & 0.05 & 0.0008 & & \\
\hline & & City College NY-CUNY & 0.08 & 0.0148 & & & $40-b$ & 48 \\
\hline & & Columbia Presbyterian Medical Center & 14.23 & 14.8107 & 0.88 & 1.4800 & $56-b$ & 6 \\
\hline & & Columbia University & 0.64 & 48.0045 & 13.10 & 501.1000 & $100-b$ & 80 \\
\hline & & Haskins Laboratories of Pace University & $*$ & 0.0383 & & & 15.3-b & 120 \\
\hline & & Hunter College - CUNY & 0.39 & 0.4586 & & & & 6 \\
\hline & & Memorial Sloan-Kettering Cancer Center & 5.62 & 4.8600 & 16.80 & 1.8981 & $560-b$ & 6 \\
\hline & & Mt. Sinai Medical Center & 1.05 & 0.6114 & & & 28-b & 18 \\
\hline & & $\begin{array}{l}\text { New York City Department of Health } \\
\text { Bureau of Laboratories }\end{array}$ & & & 0.42 & 3.7000 & 63-b & 26 \\
\hline
\end{tabular}

1 Eastman Kodak reported normal accumulation of waste pending sufficient volume for disposal.

2 The New York Office submits a single report reflecting the activities and volumes from all Bell Atlantic facilities in the State.

† The letter " $b$ " following an entry in the Storage Capacity column indicates storage capacity that includes both storage pending disposal in a licensed LLRW disposal facility and storage for decay. Entries without a " $\mathrm{b}$ " indicate capacity for storage pending disposal in a licensed LLRW disposal facility.

* Less than 0.1 cubic meter or $0.0001 \mathrm{GBq}$. 


\begin{tabular}{|c|c|c|c|c|c|c|c|c|}
\hline \multirow{24}{*}{ 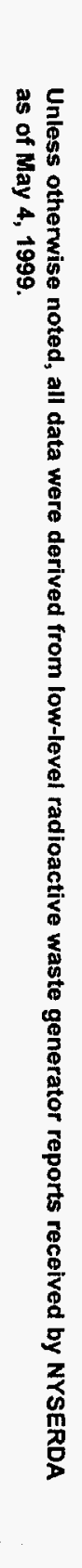 } & County & Facility \& Location & $\begin{array}{c}\text { Volume } \\
\text { Disposed } \\
\text { of }\left(\mathbf{m}^{3}\right)\end{array}$ & $\begin{array}{c}\text { Activity } \\
\text { Disposed of } \\
\text { (GBq) }\end{array}$ & $\begin{array}{c}\text { Volume } \\
\text { Stored } \\
\left(\mathbf{m}^{3}\right) \\
\end{array}$ & $\begin{array}{l}\text { Activity } \\
\text { Stored } \\
(\mathrm{GBq}) \\
\end{array}$ & $\begin{array}{c}\text { Storage } \\
\text { Capacity } \\
\left(\mathbf{m}^{3}\right) \dagger \\
\end{array}$ & $\begin{array}{l}\text { Storage Time } \\
\text { (months) }\end{array}$ \\
\hline & \multirow[t]{5}{*}{ New York } & $\begin{array}{l}\text { New York Hospital } \\
\text { Cornell Medical Center }\end{array}$ & 2.34 & 1.5307 & & & 7.7-b & 6 \\
\hline & & New York University - Lanza Laboratories & 0.04 & 0.0300 & & & & \\
\hline & & New York University Medical Center/ Bellevue Hospital & 0.06 & 0.1200 & & & & \\
\hline & & Public Health Research Institute & & & 0.63 & 0.4900 & $8-\mathrm{b}$ & 200 \\
\hline & & Rockefeller University & 1.27 & 3.6980 & 2.90 & 7.2170 & 58-b & $\geq 60$ \\
\hline & Niagara & Niagara University & 0.21 & $*$ & & & & \\
\hline & Oneida & Hamilton College, Clinton & & & 0.75 & 9.4123 & $34-\mathrm{b}$ & 500 \\
\hline & \multirow[t]{3}{*}{ Onondaga } & Bristol-Myers Squibb, East Syracuse & 0.21 & 0.1495 & & & $10.7-\mathrm{b}$ & 50 \\
\hline & & SUNY Health Science Center, Syracuse & 0.42 & 6.5453 & & & $135-b$ & 60 \\
\hline & & Syracuse University & & & & & $18-b$ & $\geq 240$ \\
\hline & \multirow[t]{3}{*}{ Oswego } & James A. FitzPatrick Nuclear Power Plant, Lycoming & 92.22 & 9500.1127 & 38.38 & 1743.8610 & 1204 & 63 \\
\hline & & Nine Mile Point Nuclear Station, Unit 1, Scriba & 55.53 & 1893710.1290 & 68.31 & 5243.0869 & 4360 & 236 \\
\hline & & Nine Mile Point Nuclear Station, Unit 2, Scriba & 16.93 & 7309.2845 & 128.87 & 23534.0037 & 4360 & 180 \\
\hline & Putnam & Orentreich Foundation, Cold Spring-on-Hudson & & & 0.26 & 0.1191 & $27.5-\mathrm{b}$ & 24 \\
\hline & \multirow[t]{2}{*}{ Queens } & Long Island Jewish Medical Center, New Hyde Park & 0.21 & 1.6939 & & & $14-b$ & 120 \\
\hline & & Queens College - CUNY, Flushing & & & 0.81 & 0.3091 & $57.1-\mathrm{b}$ & 36 \\
\hline & \multirow[t]{3}{*}{ Rensselaer } & Coromed, Inc., Troy & & & 0.16 & 0.1224 & $11.2-\mathrm{b}$ & 12 \\
\hline & & Rensselaer Polytechnic Institute, Troy & & & 15.00 & 4.0948 & $1000-b$ & 60 \\
\hline & & Virogenetics Corporation, Troy & 2.10 & 0.2634 & 1.00 & 0.1036 & $13.6-\mathrm{b}$ & 60 \\
\hline & \multirow[t]{2}{*}{$\begin{array}{l}\text { Richmond } \\
\text { (Staten Island) }\end{array}$} & $\begin{array}{l}\text { New York State Institute for Basic Research in } \\
\text { Developmental Disabilities }\end{array}$ & & & & & 1.3 & 12 \\
\hline & & St. Vincent's Medical Center & & & 0.42 & 0.0600 & $2.5-b$ & 60 \\
\hline & \multirow[t]{2}{*}{ Rockland } & Champion International Corporation, West Nyack & & & & & & \\
\hline & & ICN East, Inc., Diagnostics Division, Orangeburg & 0.08 & 0.9139 & 3.51 & 0.2433 & $72.8-b$ & 60 \\
\hline
\end{tabular}

$\uparrow$ The letter " $b$ " following an entry in the Storage Capacity column indicates storage capacity that includes both storage pending disposal in a licensed LLRW disposal facility and storage for decay. Entries without a " $b$ " indicate capacity for storage pending disposal in a licensed LLRW disposal facility.

* Less than 0.1 cubic meter or $0.0001 \mathrm{GBq}$. 


\begin{tabular}{|c|c|c|c|c|c|c|c|c|}
\hline \multirow{21}{*}{ 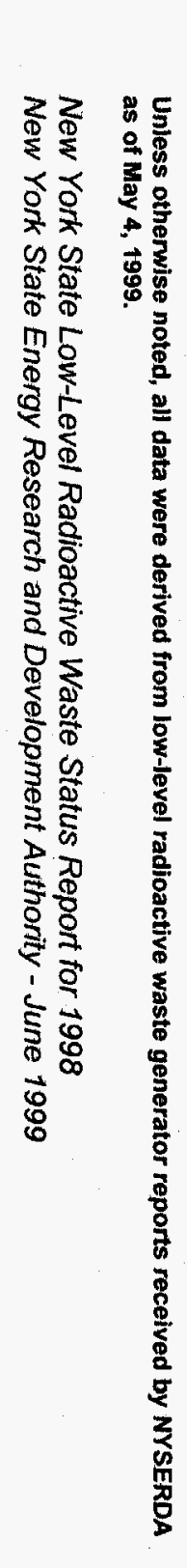 } & County & Facility \& Location & $\begin{array}{c}\text { Volume } \\
\text { Disposed } \\
\text { of }\left(\mathrm{m}^{3}\right) \\
\end{array}$ & $\begin{array}{c}\text { Activity } \\
\text { Disposed of } \\
\text { (GBq) }\end{array}$ & $\begin{array}{c}\text { Volume } \\
\text { Stored } \\
\left(\mathrm{m}^{3}\right) \\
\end{array}$ & $\begin{array}{l}\text { Activity } \\
\text { Stored } \\
\text { (GBq) } \\
\end{array}$ & $\begin{array}{c}\text { Storage } \\
\text { Capacity } \\
\left(\mathbf{m}^{3}\right) \dagger\end{array}$ & $\begin{array}{l}\text { Storage Time } \\
\text { (months) }\end{array}$ \\
\hline & \multirow[t]{3}{*}{ Rockland } & Nathan Kline Institute, Orangeburg & 0.06 & 0.3740 & & & 45-b & 84 \\
\hline & & Self-Powered Lighting, West Nyack & & & 0.53 & 176.7310 & 4.2 & 60 \\
\hline & & Wyeth Ayerst Research, Pearl River & 0.94 & 55.1840 & & & $340-\mathrm{b}$ & 24 \\
\hline & Schenectady & General Electric Corporate $R \& D$, Niskayuna & 1.06 & 1.0981 & 0.21 & 2.3852 & 5 & 36 \\
\hline & Seneca & Seneca Army Depot, Romulus & 2.90 & 0.0016 & & & 680 & 24 \\
\hline & St. Lawrence & Clarkson University, Potsdam & & & 0.10 & 0.2080 & $10-b$ & 120 \\
\hline & Suffolk & SUNY-Stony Brook & 3.55 & 1.8305 & 1.68 & 2.0000 & 63-b & 48 \\
\hline & \multirow[t]{2}{*}{ Tompkins } & $\begin{array}{l}\text { Cornell University, Environmental Health \& Safety, } \\
\text { Ithaca }\end{array}$ & 0.74 & 3.9351 & & & $41-b$ & 28 \\
\hline & & Ithaca College & & & 0.30 & 0.3288 & $12-b$ & 120 \\
\hline & Ulster & New York City DEP - Ben Nesin Laboratory, Shokan & & & & & & 12 \\
\hline & Wayne & R.E. Ginna Nuclear Power Plant, Ontario & 15.62 & 7319.1620 & 4.42 & 1.7300 & 560 & 60 \\
\hline & \multirow[t]{9}{*}{ Westchester } & $\begin{array}{l}\text { American Health Foundation } \\
\text { Naylor Dana Institute, Valhalla }\end{array}$ & 5.88 & 15.0810 & & & $5.2-b$ & $<6$ \\
\hline & & $\begin{array}{l}\text { Consolidated Edison Co. of New York, Inc., Indian } \\
\text { Point \#2, Buchanan }\end{array}$ & 58.18 & 97480.391 & & & 6000 & 60 \\
\hline & & Immunotherapy, Inc., Tarrytown & & & 0.29 & 1.9906 & $0.5-b$ & 14 \\
\hline & & Medi-Ray, Tuckahoe & 7.50 & 0.0185 & 7.50 & 0.0185 & $120-\mathrm{b}$ & 60 \\
\hline & & $\begin{array}{l}\text { New York Hospital/Cornell Medical Center, } \\
\text { White Plains }\end{array}$ & 0.42 & 0.0037 & & & $16.5-\mathrm{b}$ & 120 \\
\hline & & New York City DEP - Kensico Laboratory, Valhalla & 0.02 & 0.5550 & & & & 12 \\
\hline & & New York Power Authority, Indian Point \#3, Buchanan & 34.43 & 7540.3976 & & & 1699 & 360 \\
\hline & & Regeneron Pharmaceuticals, Inc., Tarrytown & 0.05 & 0.2590 & & & 29.9-b & 70 \\
\hline & & $\begin{array}{l}\text { The W.M. Burke Medical Research Institute, } \\
\text { White Plains }\end{array}$ & & & & & 42-b & 120 \\
\hline
\end{tabular}

\footnotetext{
$\uparrow$ The letter "b" following an entry in the Storage Capacity column indicates storage capacity that includes both storage pending disposal in a licensed LLRW disposal facility and storage for decay. Entries without a " $b$ " indicate capacity for storage pending disposal in a licensed LLRW disposal facility.

* Less than 0.1 cubic meter or $0.0001 \mathrm{GBq}$.
} 


\section{Table 4-b: Generators Reporting Only Storage for Decay}

\author{
Albany \\ Capital Cardiology Associates, P.C., Albany \\ Capital District Endocrine Associates, Delmar \\ Capital Region Cardiology Associates, Albany \\ Empire Isotopes, LLC, Albany \\ Siena College, Loudonville \\ St. Peter's Hospital, Albany \\ Syncor International Corporation, Albany

\section{Bronx} \\ Jacobi Medical Center \\ Laboratory for Plant Morphogenesis \\ Lehman College, CUNY \\ Montefiore Medical Center \\ Our Lady of Mercy Medical Center \\ Saint Barnabas Hospital \\ Syncor Internation Corporation \\ University Diagnostic Medical Imaging \\ Westchester Square Medical Center

\section{Broome} \\ Oakdale Medical Center, Johnson City \\ Our Lady of Lourdes Memorial Hospital, \\ Binghamton \\ United Health Services Hospitals - \\ Binghamton General Hospital \\ United Health Services Hospitals - Wilson Medical \\ Center, Johnson City
}

Cayuga

Wells College, Aurora

\section{Chatauqua}

Lake Shore Health Care Center, Irving

SUNY-Fredonia

\section{Chemung}

Arnot Ogden Medical Center, Elmira

Guthrie Clinic, Big Flats Office, Horseheads

St. Joseph's Hospital, Elmira

\section{Clinton}

CVPH Medical Center, Plattsburgh

\section{Cortland}

Commons Cardiac Evaluation Centre, Cortland

Delaware

Delaware Valley Hospital, Walton

The Hospital, Sydney

\section{Dutchess}

Bard College, Annandale on Hudson

Clinical Endocrine Laboratory, Poughkeepsie

DRA Imaging, Poughkeepsie

Hudson Valley Heart Center, Poughkeepsie

St. Francis' Hospital, Poughkeepsie

V.A. Hudson Valley Health Care Systems, Castle Point

Erie

Buffalo Cardiology and Pulmonary Associates

Buffalo General Hospital

Buffalo Medical Group, P.C.

Buffalo Medical Group, P.C., Williamsville

Cardiology Group of Western New York, Williamsville

Central Radiopharmaceutical Services, Buffalo

Erie County Medical Center, Buffalo

Kenmore Mercy Hospital, Kenmore

Life Technologies, Grand Island

Mercy Hospital, Buffalo

Millard Fillmore Hospital, Buffalo

Millard Fillmore Hospitals, Williamsville

Millard Fillmore Suburban Hospital, Williamsville

Roswell Park Cancer Institute Corporation, Buffalo

Sheehan Memorial Hospital, Buffalo

Sisters of Charity Hospital, Buffalo

Syncor International Corporation, Cheektowaga

Essex

Adirondack Biomedical Research Institute,

Lake Placid

Franklin

Adirondack Medical Center, Saranac Lake

Trudeau Institute, Inc., Saranac Lake

Genesee

Genesee Memorial Hospital, Batavia

Herkimer

Little Falls Hospital

Jefferson

James E. Willis, M.D., P.C., Watertown

Mirza M. Ashraf, M.D., Carthage

Samaritan Medical Center, Watertown

Kings (Brooklyn)

Bay Imaging

Brooklyn Medical Imaging Center 
Brooklyn Nuclear Services

Cardio Lab, Inc.

Brooklyn College - CUNY

Diagnostic Cardiology Associates, P.C.

Family Health Care and Cardiac Center

Long Island College Hospital

Lutheran Medical Center

Mark Novick, M.D.

Med Spect Nuclear Imaging, P.C.

New York Methodist Hospital

The Brooklyn Hospital Center

Universal Diagnostic Laboratories, Inc.

U.S. FDA - Northeast Regional Laboratory

Wyckoff Heights Medical Center

\section{Livingston}

Nicholas Noyes Memorial Hospital, Dansville

\section{Madison}

Colgate University, Hamilton

Oneida Healthcare Center, Oneida

\section{Monroe}

Lakeside Memorial Hospital, Brockport

Monroe County Medical Examiner, Rochester

Park Ridge Hospital, Rochester

Rochester Cardiopulmonary Group, P.C.

Rochester General Hospital

St. Mary's Hospital, Rochester

Syncor International Corporation, Rochester

Wyeth Lederle Vaccines and Pediatrics,

West Henrietta

\section{Montgomery}

Amsterdam Memorial Hospital

St. Mary's Hospital, Amsterdam

\section{Nassau}

Advanced Medical Imaging of Long Island, Great Neck

Bethpage Medical Laboratory

Cardiovascular Diagnostic Services, Plainview

Cardiovascular Medical Associates; Garden City

Day-Op Center of Long Island, P.C., Mineola

Endocrine and Diabetes Associates of Long Island,

Rockville Centre

Franklin Hospital Medical Center, Valley Stream

Grappell and Walker, M.D., P.C., Plainview

Great Neck Imaging

Heart Diagnostic Imaging, Great Neck

Hempstead General Hospital

Howard Heimowitz, M.D., Syosset

Long Beach Medical Center
Long Island Cardiovascular Imaging Consultants,

P.C., Great Neck

Mallinckrodt, Inc., Hicksville

Manhasset Diagnostic Imaging

Mercy Medical Center, Rockville Centre

Nassau Cardiac Imaging, Valley Stream

Nassau County Medical Examiner's Office,

East Meadow

Nassau Radiologic Group, P.C., Garden City

Nassau Radiological Group, Manhasset

North Shore Cardiac Imaging, P.C., New Hyde Park

North Shore University Hospital at Glen Cove

Nycomed Amersham, Port Washington

South Shore Diagnostic Heart Center, Massapequa

South Shore Heart Associates, P.C., Rockville Centre

SUNY College at Old Westbury

Weber and Bloom, P. C., Smithtown

\section{New York}

Advanced Fertility Services, P.C.

Bendiner and Schlesinger, Inc.

Beth Israel Medical Center

Dr. Grunter and Dr. Stoll, M.D., P.C.

Dr. Jacob Lichy and Dr. Thomas Kolb

East Side Physicians, P.C.

Gramercy Cardiac Diagnostic Services

Harlem Hospital Center

Lenox Hill Hospital

Lenox Hill Radiology and Medical Imaging

Associates

Metropolitan Hospital Center

New York Blood Center

New York University

Orentreich Medical Group, LLP (Lab)

Park Avenue Radiologists, P.C.

St. Luke's Roosevelt Hospital Center

St. Luke's Roosevelt Hospital

West End MRI Medical Associates, P.C.

Niagara

Mount St. Mary's Hospital, Lewiston

Oneida

Faxton Hospital, Utica

St. Elizabeth Hospital, Utica

St. Luke's Memorial Hospital, Utica

\section{Onondaga}

Community General Hospital, Syracuse

Crouse Hospital, Syracuse

CV Group, LLC, Fayetteville

CV Group, LLC - North Medical Cardiovascular

Heart Care Center, Liverpool 
Onondaga Hill Cardiovascular Group, Liverpool St. Joseph's Hospital Health Center, Syracuse Syncor International Corporation, Syracuse

\section{Ontario}

Clifton Springs Hospital and Clinic

F.F. Thompson Hospital, Canandaigua

Geneva General Hospital

\section{Orange}

Hudson Valley Heart Center, New Windsor Mid-Hudson Cardiology, P.C., New Windsor Nuclear Imaging Systems, Inc., Newburgh

St. Anthony's Hospital, Warwick

St. Luke's Hospital, Newburgh

Syncor International Corporation, Newburgh

The Cornwall Hospital

Wallkill Radiology Associates, P.C., Middletown

\section{Orleans}

Medina Memorial Hospital

\section{Otsego}

A.O. Fox Hospital, Oneonta

Bassett Healthcare, Cooperstown

Hartwick College, Oneonta

\section{Putnam}

New England Equine Practice, P.C., Brewster

\section{Queens}

Elmhurst Hospital Center (City Hospital) EMA Medical Laboratory, Inc., Ridgewood Gramercy Cardiac Diagnostic Services, Forest Hills Hillcrest Radiology Associates, P.C., Jamaica North Shore University Hospital at Forest Hills Parkway Hospital, Forest Hills Peninsula Hospital Center, Far Rockaway Richmond Hill Nuclear Imaging Vascular Diagnostic Associates, P.C., Flushing Western Queens Community Hospital, Long Island City

York College - CUNY, Jamaica

\section{Rensselaer}

Regeneron Pharmaceuticals, Inc., Rensselaer

\section{Richmond (Staten Island)}

Doctors' Hospital of Staten Island

Regional Radiology

Young F. Eng, M.D.

\section{Rockland}

Dr. Noah Weg and Associates, Suffern
Helen Hayes Hospital, West Haverstraw Mid-Rockland Imaging Associates, New City

\author{
Saratoga \\ Saratoga Cardiology Associates, P.C., \\ Saratoga Springs \\ Saratoga Hospital, Saratoga Springs \\ Skidmore College, Saratoga Springs \\ Schenectady \\ Cardiology Associates of Schenectady \\ Ellis Hospital, Schenectady \\ St. Clare's Hospital, Schenectady
}

\section{St. Lawrence}

Cardiac Fitness, Inc. at E.J. Noble of Gouveneur St. Lawrence University, Canton

\section{Steuben}

Corning Hospital

St. James Mercy Hospital, Hornell

\section{Suffolk}

Amityville Heart Center

Amplicon Corporation, East Setauket

BAB Radiological, Bay Shore

Brookhaven Memorial Medical Center

Central Suffolk Hospital, Riverhead

Department of Veterans' Affairs Medical Center, Northport

East End Cardiology, P.C., Riverhead Eastern Long Island Hosptial, Greenport

Eastern Suffolk Cardiology, Riverhead

Gary E. Veit, M.D., Bay Shore

Good Samaritan Hosptial, West Islip

Huntington Hospital

Huntington Medical Group, P.C.

Island Cardiovascular II, Port Jefferson

John Ruisi, M.D., Amityville

Long Island Medical Diagnostic Imaging, East Islip

Neometrics, Inc., East Northport

North Fork Radiology, Riverhead

North Shore Hematology and Oncology Associates, East Setauket

North Suffolk Cardiology Associates, Stony Brook South Bay Cardiovascular Associates, Bay Shore

South Shore Cardiologists, West Islip

Southampton Hospital

Southside Hospital, Bay Shore

St. John's Episcopal Hospital, Smithtown

Stony Brook Medical Imaging, P.C.

Suffolk Heart Group, P.C., Bay Shore

Sunrise Medical Laboratories, Hauppauge

Suffolk Nuclear Imaging, East Patchogue 
The Huntington Heart Center

United Biomedical, Inc., Hauppauge

UTC International, Farmingdale

\section{Tompkins}

Cayuga Medical Center at Ithaca

\section{Ulster}

Benedictine Hospital, Kingston

Ellenville Community Hospital

Kingston Diagnostic Center

SUNY - New Paltz

The Kingston Hospital

\section{Warren}

Glens Falls Hospital

\section{Wayne}

ViaHealth of Wayne - Newark Campus

\section{Westchester}

Cardiology Consultants of Westchester, Hawthorne

DOCS Physicians Affiliated with Beth Israel

Hospital, Yonkers

Emisphere Technologies, Inc., Tarrytown

Hudson Valley Heart Center, Cortlandt Manor

Mount Vernon Hospital

Northern Westchester Hospital, Mt. Kisco

Phelps Memorial Hospital, North Tarrytown

Polymedco, Inc., Cortlandt Manor

Progenics Pharmaceuticals, Inc., Tarrytown

Rye Radiology, Ryebrook

Sohn, M.D. Laboratory, Larchmont

Sound Shore Medical Center of Westchester,

New Rochelle

St. Agnes Hospital, White Plains

Westchester County Department of Laboratories and

Research, Valhalla

Westchester Heart Specialists, New Rochelle

Westchester Medical Center, Valhalla

White Plains Hospital Center 


\section{CONVERSIONS FOR UNITS}

The standardized measurement units used in science and technology today are known as the metric system. These metric or SI system (Système International d'Unités) units have been incorporated in the NRC's Uniform Waste Manifest. This manifest has been adopted by the licensed LLRW disposal facility in Barnwell, South Carolina. Use of the NRC Uniform Waste Manifest became mandatory on March 1, 1998.

Volume and activity are presented in cubic meters and gigabecquerels $(\mathrm{GBq})$ or megabecquerels $(\mathrm{Mbq})$. These units have been adopted for this report to be consistent with the uniform national LLRW manifest requirements. Some conversions for SI units to the previously used units of cubic feet and curies are in the following tables.

\begin{tabular}{|c|c|c|c|c|}
\hline \multicolumn{5}{|c|}{ CONVERSIONS FOR UNITS } \\
\hline Quantity & SI Unit & $\begin{array}{l}\text { Previously } \\
\text { Used Unit } \\
\end{array}$ & $\begin{array}{c}\text { Value of } \\
\text { Conventional Unit in } \\
\text { SI Units } \\
\end{array}$ & Conversion Factors \\
\hline Activity & $\begin{array}{l}\text { Gigabecquerel }(\mathrm{GBq}) \\
\text { Megabecquerel }(\mathrm{MBq})\end{array}$ & Curie (Ci) & $\begin{array}{l}1 \mathrm{Ci}=37 \mathrm{GBq} \\
1 \mathrm{Ci}=37,000 \mathrm{MBq}\end{array}$ & $\begin{array}{l}\mathrm{Ci} \times 37=\mathrm{GBq} \\
\mathrm{Ci} \times 37,000=\mathrm{MBq} \\
\mathrm{GBq} \div 37=\mathrm{Ci} \\
\mathrm{MBq} \div 37,000=\mathrm{Ci}\end{array}$ \\
\hline Volume & cubic meters $\left(\mathrm{m}^{3}\right)$ & cubic feet $\left(\mathrm{ft}^{3}\right)$ & $1 \mathrm{ft}^{3}=0.028 \mathrm{~m}^{3}$ & $\begin{array}{l}\mathrm{ft}^{3} \times 0.028=\mathrm{m}^{3} \\
\mathrm{~m}^{3} \times 35.31=\mathrm{ft}^{3}\end{array}$ \\
\hline
\end{tabular}

\begin{tabular}{|c|c|r|}
\hline \multicolumn{3}{|c|}{ Activity Conversions } \\
\hline \hline $\mathbf{m C i}$ & $\mathbf{M B q}$ & \multicolumn{1}{|c|}{$\mathbf{G B q}$} \\
\hline 500 & 18,500 & 18.500 \\
\hline 200 & 7,400 & 7.400 \\
\hline 100 & 3,700 & 3.700 \\
\hline 50 & 1,850 & 1.850 \\
\hline 20 & 740 & 0.740 \\
\hline 10 & 370 & 0.370 \\
\hline 5 & 185 & 0.185 \\
\hline 2 & 74 & 0.074 \\
\hline 1 & 37 & 0.037 \\
\hline
\end{tabular}

\begin{tabular}{||c|c|}
\hline \multicolumn{2}{|c|}{ Volume Conversions } \\
\hline \hline $\mathbf{f t}^{3}$ & $\mathbf{m}^{\mathbf{3}}$ \\
\hline 11.9 (89 gal. drum) & 0.33 \\
\hline 11.1 (83 gal. drum) & 0.31 \\
\hline 7.5 (55 gal. drum) & 0.21 \\
\hline 4.01 (30 gal. drum) & 0.11 \\
\hline 0.67 (5 gal. pail) & 0.019 \\
\hline & \\
\hline & \\
\hline & \\
\hline & \\
\hline
\end{tabular}

